This item was submitted to Loughborough's Research Repository by the author.

Items in Figshare are protected by copyright, with all rights reserved, unless otherwise indicated.

\title{
Toward an understanding of electronic excitation energies beyond the molecular orbital picture
}

\section{PLEASE CITE THE PUBLISHED VERSION}

https://doi.org/10.1039/D0CP00369G

\section{PUBLISHER}

Royal Society of Chemistry (RSC)

\section{VERSION}

AM (Accepted Manuscript)

\section{PUBLISHER STATEMENT}

This paper was accepted for publication in the journal Physical Chemistry Chemical Physics and the definitive published version is available at https://doi.org/10.1039/D0CP00369G.

\section{LICENCE}

CC BY-NC-ND 4.0

\section{REPOSITORY RECORD}

Kimber, Patrick, and Felix Plasser. 2020. "Toward an Understanding of Electronic Excitation Energies Beyond the Molecular Orbital Picture". Loughborough University. https://hdl.handle.net/2134/11913378.v1. 

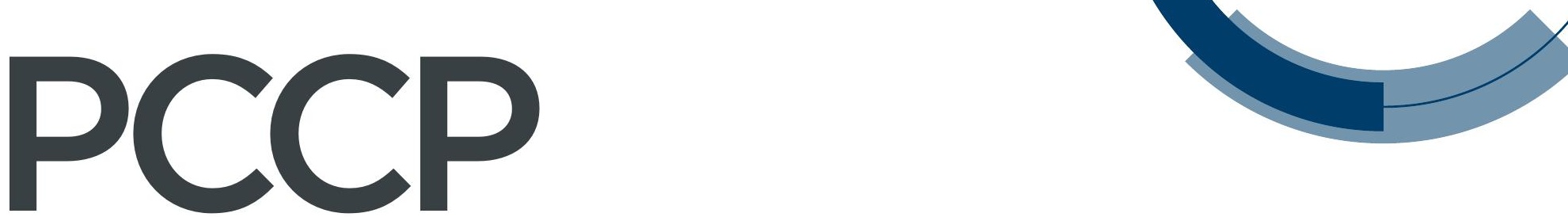

Physical Chemistry Chemical Physics

\section{Accepted Manuscript}

This article can be cited before page numbers have been issued, to do this please use: $P$. Kimber and $F$. Plasser, Phys. Chem. Chem. Phys., 2020, DOI: 10.1039/D0CP00369G.
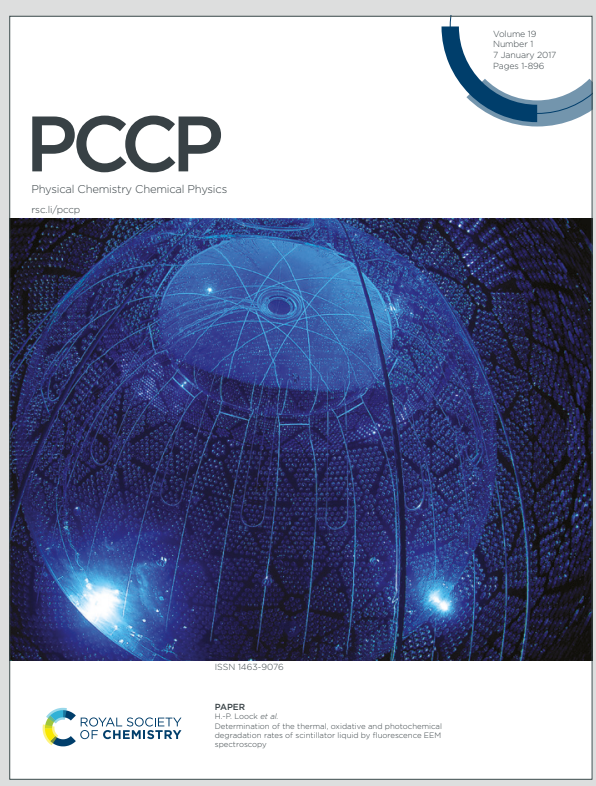

This is an Accepted Manuscript, which has been through the Royal Society of Chemistry peer review process and has been accepted for publication.

Accepted Manuscripts are published online shortly after acceptance, before technical editing, formatting and proof reading. Using this free service, authors can make their results available to the community, in citable form, before we publish the edited article. We will replace this Accepted Manuscript with the edited and formatted Advance Article as soon as it is available.

You can find more information about Accepted Manuscripts in the Information for Authors.

Please note that technical editing may introduce minor changes to the text and/or graphics, which may alter content. The journal's standard Terms \& Conditions and the Ethical guidelines still apply. In no event shall the Royal Society of Chemistry be held responsible for any errors or omissions in this Accepted Manuscript or any consequences arising from the use of any information it contains. 


\section{PCCP}

\section{ARTICLE TYPE}

Cite this: DOI: $00.0000 / x x x x x x x x x x$

\section{Toward an Understanding of Electronic Excitation En- ergies Beyond the Molecular Orbital Picture ${ }^{\dagger}$}

\author{
Patrick Kimber, ${ }^{a}$ Felix Plasser ${ }^{* a}$
}

\section{Received Date}

Accepted Date

DOI: $00.0000 / \operatorname{xxxxxxxxxx}$

\section{Introduction}

The design of molecular systems with specific absorption and emission properties is a major theme in modern chemistry with relevance to lighting, 1.3 photovoltaics, 4 chemical synthesis, and to more specific uses such as photoinitiators, 7 fluorescent probes, $\frac{89}{}$ and sensors. $10[11$ Most commonly new chromophores are designed based on the energies and shapes of their frontier orbitals, which have proven to be an intuitive and powerful concept for understanding electronic structure despite their purely quantum mechanical nature. Using simple rules, e.g. that electron-withdrawing groups lower orbital energies while electron-donating groups increase them, it is indeed possible to modify the energies and characters of excited states in organic chromophores ${ }^{9] 12}\left[14\right.$ and transition metal complexes. ${ }^{15 \mid 16}$ On the one hand, this success of the frontier orbital picture is an impressive account of the influence that abstract quantum mechanical concepts can have on practising chemists and has ultimately lead to a Nobel Prize in Chemistry. $17 \mid 18$ On the other hand, it is sur-

\footnotetext{
${ }^{a}$ Department of Chemistry, Loughborough University, Loughborough, LE11 3TU, U.K.; Tel: +44 1509 226946; E-mail: f.plasser@lboro.ac.uk

$\dagger$ Electronic Supplementary Information (ESI) available: frontier orbitals of uracil (S1), transition densities and their ESPs for polyacene singlet (S2) and triplet (S3) states and octatetraene singlet states (S4). See DOI: 10.1039/cXCP00000x/
}

prising that an intermediate quantity in an approximate theory in explaining to people outside the community why chemists ascribe almost prophetic power to pictures showing coloured blobs of various shapes and sizes, and the unscrupulous application of orbital energies is regularly criticised by theorists. $19, \mid 21$

Limitations of the molecular orbital (MO) picture are encountered in a variety of molecular systems used for different applications. These limitations become particularly apparent when comparing the energies of singlet and triplet states that, aside from spin coupling, are reached via the same orbital transitions. In the zero-order MO description such states would have the same energy but molecules in practical use range from almost vanishing gaps between the first singlet and triplet excited states capable of delayed fluorescence ${ }^{2 / 22}$ to cases where the singlet is twice the triplet energy to be used for singlet fission. 23.25 Some rules exist, e.g. minimising the spatial overlap between the highest occupied MO (HOMO) and the lowest unoccupied MO (LUMO) ${ }^{1 \mid 22}$ or including double excitations 26 for delayed fluorescence and exploiting diradical character in the ground state ${ }^{27-29}$ or aromaticity in the triplet state ${ }^{30}$ for singlet fission but a more comprehensive theoretical framework is not available and unwanted sideeffects are hard to exclude. $\frac{31}{3}$ More specific cases of the breakshould gain such prominence. Indeed, one is often hard-pressed 
down of the MO picture are concerned with exciton correlation in conjugated polymers, concerning linear $\sqrt{32+34}$ and cyclic 3536

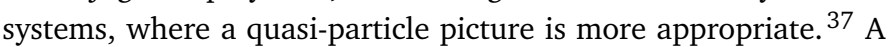
further long known,,$\sqrt{8139}$ but often neglected, problem is the differentiation between ionic and covalent states in hydrocarbons, which emerges in a valence-bond description but is hard to do within the MO picture. ${ }^{40}$ (42) $\mathrm{But}$ the limitations of the MO picture also surface in day-to-day work, e.g. when pondering why the HOMO-LUMO transition does not always give rise to the lowest excited state of a molecule.

A deeper understanding of excited-state energies could also have huge implications on the application and design of new computational excited-state methods. It is striking that already simple HOMO-LUMO transitions in conjugated hydrocarbons can be detrimental to, both, time-dependent density functional theory $\sqrt{43 \sqrt{46}}$ and multi-reference based wavefunction descriptions. ${ }^{47-51}$ More problems come into play for charge-transfer states,,$[52]$ correlated excitons, ${ }^{[53[54}$ and states with enhanced orbital relaxation. $\sqrt{55}$ Furthermore, striking differences are found between the descriptions of singlet and triplet states within TDDFT ${ }^{56 \mid 57}$ and the Bethe-Salpeter equation (BSE) ${ }^{[58}$ In all these cases it is difficult to understand the problem from the orbitals alone. It is common to use the blanket term "electron correlation" for everything that does not fit the picture but that hardly provides constructive insight.

A number of wavefunction analysis techniques have been developed for categorising excited states in a rigorous way and for automating the analysis process. Aside from basic visualisation techniques, $[59,61$ a significant effort has been spent in designing quantitative descriptors measuring a variety of properties such as charge-transfer, $37 \mid 62+65$ double excitation character, 6166667 and entanglement. 68 .71 $\mathrm{A}$ particular effort has been devoted to the task of visualising excited-state correlations using either correlation plots ${ }^{72-77}$ or a newly developed technique for visualising correlation effects in real space. ${ }^{42}$ The availability of the tools discussed above can have a substantial impact on applied studies not only through quantifying excited-state character 978879 but also through providing new insight into intricate wavefunction properties that would otherwise be hard to come by. ${ }^{[4 \mid 80]}$ However, so far wavefunction analysis is usually restricted to characterising excited states within pre-existing models based mostly on the MO picture. It is the purpose of this Perspective to investigate whether we can use the same approach, a detailed and quantitative analysis of electronic wavefunctions, to derive new qualitative insight into excitation energies. Can we derive design principles for tuning molecular excitation energies that truly go beyond the MO picture?

The strategy employed here is to, first, obtain a comprehensive understanding of the physics governing the energy of a correlated electron-hole pair using the language of and insights from modern quantum chemistry. Doing so, we identify two main interaction terms, the exchange repulsion and dynamic Coulomb attraction while also discussing two additional terms encountered in practical computations, secondary orbital relaxation and deexcitations. It is discussed in detail under what circumstances these terms are expected to play a role and which experimentally

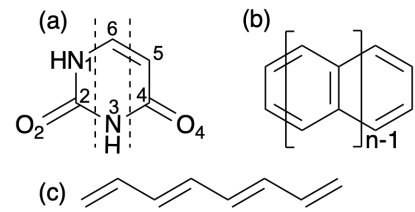

Fig. 1 Molecules investigated in this work: (a) uracil, (b) polyacenes $(n=2, \ldots, 5)$, and (c) octatetraene. For uracil, the IUPAC numbering scheme as well as the fragmentation scheme used in the wavefunction analysis are shown.

observed phenomena are based on them. Subsequently, we show how to reconstruct these contributions from realistic excited-state computations using a variety of quantum chemistry methods.

The article is structured as follows. In Sec. 2 we to discuss the theory describing the physics of a correlated electron-hole pair in some detail to show how one can move from a general energy expression in second quantisation to a quite intuitive and memorable form for the different terms. The computational details are collected in Sec. 3 . In Sec. 4 the theory is applied to three distinct molecular systems as shown in Fig. 1 to demonstrate the versatility of the developed methods. Firstly, uracil is chosen as a representative small organic molecule and the relative ordering of its singlet and triplet $\pi \pi^{*}$ and $n \pi^{*}$ states is discussed. We focus on why the exchange splitting between $n \pi^{*}$ states of heteroaromatic molecules is generally much lower than that between the $\pi \pi^{*}$ states. Next, polyacenes of different length are investigated to elucidate the reason for their large $S_{1} / T_{1}$ gaps, which underlie their propensity to undergo singlet fission. ${ }^{23}$ We conclude with a more specific issue, the emergence of plasmonic states in octatetraene. 81

\section{Theory}

The aim of this work is to develop an intuitive but also rigorous approach for understanding electronic excitation energies. A simple discussion of orbital energies can be seen as a zeroth order description of the excitation energy and we want to move to the first order here. To do so, we analyse the configuration interaction singles (CIS) model. CIS is chosen, not because it is an accurate method by itself, but because it is the formal starting point for most excited-state methods used, i.e. the CIS energies are equivalent to the lowest order coupled cluster (CCS) and algebraic diagrammatic construction (ADC(1)) energies ${ }^{82 / 83}$ and CIS possesses the same formal structure as TDDFT and BSE in the Tamm-Dancoff approximation. ${ }^{8485}$ Therefore, we argue that CIS captures the main physics of a singly excited state or, in other words, of a correlated electron-hole pair.

The purpose of this section is to provide a self-contained derivation of the CIS excitation energy and subsequently reinterpret the terms occurring as interaction terms of a correlated electron-hole pair. In this process we will make extended use of Wick's theorem ${ }^{86}$ recognizing its recent use in a related context. ${ }^{87}$ In a subsequent step, we will use a diagrammatic representation $\sqrt{88}$ of the relevant terms. Finally, we interpret the terms using various density matrices and recast the terms occurring into pictorial form. Sec. 2.1 introduces the notation used. In Sec. 2.2, we show how 
the CIS energy can be brought into a more memorable form, and the main results are reviewed in Sec. 2.3 We proceed by discussing simplifications relevant for a two-orbital model (Sec. 2.4) and conclude by discussing how to assess the validity of the model in realistic excited-state computations (Sec. 2.5).

\subsection{General Notation}

In this work we will denote the ground state wavefunction as $\left|\Psi_{0}\right\rangle$ and the excited state wavefunction as $\left|\Psi_{I}\right\rangle$. The excited state energy is given as

$$
E_{I}=\left\langle\Psi_{I}|\hat{\mathrm{H}}| \Psi_{I}\right\rangle
$$

where the Hamiltonian operator in second quantization is defined as ${ }^{89}$

$$
\hat{\mathrm{H}}=\sum_{p q} h_{p q} \hat{\mathrm{a}}_{p}^{\dagger} \hat{\mathrm{a}}_{q}+\sum_{p q r s}(p q \mid r s) \hat{\mathrm{a}}_{p}^{\dagger} \hat{\mathrm{a}}_{r}^{\dagger} \hat{\mathrm{a}}_{s} \hat{\mathrm{a}}_{q}
$$

Here, $h_{p q}$ represents a matrix element of the core Hamiltonian, i.e. the kinetic energy and the electron-nucleus attraction, and $\hat{a}_{p}^{\dagger}$ and $\hat{\mathrm{a}}_{q}$ refer to the creation and annihilation operators pertaining to molecular orbitals (MOs) $\phi_{p}$ and $\phi_{q}$. The two-electron repulsion integrals, written in "charge-cloud" notation, are defined as

$$
(p q \mid r s)=\iint \frac{\phi_{p}\left(r_{1}\right) \phi_{q}\left(r_{1}\right) \phi_{r}\left(r_{2}\right) \phi_{s}\left(r_{2}\right)}{r_{12}} \mathrm{~d} r_{1} \mathrm{~d} r_{2} .
$$

To characterise the excited state wavefunction, we will make use of the one-electron transition density matrix (1TDM), which is given as

$$
\begin{gathered}
\gamma_{0 I}\left(r_{h}, r_{e}\right)=\sum_{p q} \gamma_{p q}^{0 I} \phi_{p}\left(r_{h}\right) \phi_{q}\left(r_{e}\right) \\
\gamma_{p q}^{0 I}=\left\langle\Psi_{0}\left|\hat{\mathrm{a}}_{p}^{\dagger} \hat{\mathrm{a}}_{q}\right| \Psi_{I}\right\rangle
\end{gathered}
$$

where the coordinates $r_{h}$ and $r_{e}$ are interpreted as the positions of the excitation hole and the excited electron, respectively. $37 / 61$ Note, that we use the symbol $\gamma_{0 I}$ for the 1TDM in real space and $\gamma_{p q}^{0 I}$ for its matrix elements with respect to an orthonormal MO basis. After applying a singular value decomposition, the 1TDM can be represented in a more compact diagonal form called the natural transition orbital (NTO) $\sqrt[60 / 76]{6}$ representation

$$
\gamma_{0 I}\left(r_{h}, r_{e}\right)=\sum_{t} \sqrt{\lambda_{t}} \psi_{t}^{h}\left(r_{h}\right) \psi_{t}^{e}\left(r_{e}\right) .
$$

Here, $\psi_{t}^{h}$ and $\psi_{t}^{e}$ are the NTOs representing the hole and electron and $\lambda_{t}$ is the amplitude of the transition. Aside from providing rigorous and compact pictorial representations, the NTO decomposition provides a natural measure of the multiconfigurational character of the excited state independently of the orbital representation. In the case, of a monoconfigurational state that can be completely described by a transition between two orbitals, we have

$$
\lambda_{1}=1 \quad \lambda_{2}=\lambda_{3}=\ldots=0
$$

and the 1TDM factorises into a simple product

$$
\gamma_{0 I}\left(r_{h}, r_{e}\right)=\psi^{h}\left(r_{h}\right) \psi^{e}\left(r_{e}\right)
$$

of two, possibly non-orthogonal, orbitals. Conversely, if several NTO amplitudes are non-vanishing, then the 1TDM will encode non-trivial correlation and interference effects between the different orbitals. Therefore, the number of non-vanishing NTO amplitudes can be seen as a fundamental property of an excited

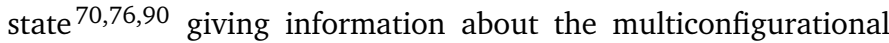
character. We have previously suggested based on heuristic reasoning to count the number of configurations via the NTO participation ratio 76

$$
\mathrm{PR}_{\mathrm{NTO}}=\frac{\left[\operatorname{tr}\left(\gamma_{0 I} \gamma_{0 I}^{\mathrm{T}}\right)\right]^{2}}{\operatorname{tr}\left(\gamma_{0 I} \gamma_{0 I}^{\mathrm{T}}\right)^{2}}=\frac{\left[\sum_{t} \lambda_{t}\right]^{2}}{\sum_{t} \lambda_{t}^{2}}
$$

which is equivalent 70 to the collectivity number defined by Luzanov and co-workers. ${ }^{90}$ More formally speaking, we can interpret the multiconfigurational character in terms of entanglement of the electron and hole quasiparticles, and compute a number of entangled states

$$
Z_{H E}=2^{-\sum_{t} \lambda_{t} \log _{2} \lambda_{t}}
$$

via the von Neumann entropy.70 ${ }^{70}$ Both measures are exactly 1 if Eq. (7) holds and they are higher for multiconfigurational states. In the following, we will use $\mathrm{PR}_{\mathrm{NTO}}$, considering that this has already found various applications in the literature $\frac{36 / 80|91| 92}{d e}$ spite its ad hoc nature.

The transition density is defined as the diagonal part of the $1 \mathrm{TDM}$

$$
\rho_{0 I}(r)=\gamma_{0 I}(r, r)
$$

It is the function obtained by multiplying the occupied and virtual orbitals forming the individual excitation contributions. In the electron-hole picture it corresponds to cases where the electron and hole are at the same point in space. In addition, we define the density matrices for the hole and electron as $\underline{61}$

$$
\begin{aligned}
& \gamma_{h}\left(r_{h}, r_{h}^{\prime}\right)=\int \gamma_{0 I}\left(r_{h}, r_{e}\right) \gamma_{0 I}\left(r_{h}^{\prime}, r_{e}\right) \mathrm{d} r_{e} \\
& \gamma_{e}\left(r_{e}, r_{e}^{\prime}\right)=\int \gamma_{0 I}\left(r_{h}, r_{e}\right) \gamma_{0 I}\left(r_{h}, r_{e}^{\prime}\right) \mathrm{d} r_{h}
\end{aligned}
$$

And their associated densities $\rho_{h / e}$ are defined in analogy to Eq. (11). The hole and electron densities have the intuitive interpretations as the areas in space where the excited electron comes from and where it goes to. The transition density has no such simple interpretation but has, nonetheless, crucial implications on the energetics of an excited state as explored below. In addition, the transition density is the decisive quantity for determining how strongly an electronic transition interacts with light, as determined via its dipole moment, the so-called transition dipole moment defined as

$$
\vec{\mu}_{0 I}=\left\langle\Psi_{0}|\vec{x}| \Psi_{I}\right\rangle=\int \rho_{0 I}(r) \vec{x} \mathrm{~d} r
$$

where $\vec{x}$ is the position operator. Consequently, it affects the related oscillator strength, given in atomic units as

$$
f=\frac{2 \Delta E}{3}\left|\vec{\mu}_{0 I}\right|^{2}
$$


where $\Delta E=E_{I}-E_{0}$ is the excitation energy. Finally, we define the ground-state density matrix $\gamma_{0}$ in analogy to Eq. (5) but setting $I=0$.

In many cases, it is of interest to analyse the two-body function $\gamma_{0 I}\left(r_{h}, r_{e}\right)$ in more detail to obtain information about correlations between the electron and hole quasiparticles. Initially, we have suggested following others ${ }^{74 \mid 90}$ to do so by computing the charge transfer numbers

$$
\int_{A} \int_{B}\left|\gamma_{0 I}\left(r_{h}, r_{e}\right)\right|^{2} \mathrm{~d} r_{e} \mathrm{~d} r_{h}
$$

which measure the mutual probability that the hole is on a fragment $A$ and the electron on fragment $B . \frac{61}{6}$ As a more intuitive route one of us has recently suggested the computation of conditional electron densities. $\frac{42}{42}$ For this purpose, the hole is constrained to a specific fragment $A$ and the associated conditional electron density is computed as

$$
\rho_{e}^{A}\left(r_{e}\right)=\int_{A}\left|\gamma_{0 I}\left(r_{h}, r_{e}\right)\right|^{2} \mathrm{~d} r_{h}
$$

Computing conditional densities for varying fragments allows a real space representation of correlations between the electron and hole. 42

Below, we will show that the CIS energy can be brought into a memorable and quite intuitive form if it is expressed in terms of various types of density matrices. For this purpose, we need to define some more pieces of notation. We use the common notation

$$
\operatorname{tr}\left(\gamma_{A} \mathbf{M}\right)=\sum_{p q} \gamma_{p q}^{A} M_{p q}
$$

to denote the contraction of a one-electron operator represented by the matrix $\mathbf{M}$ with a density matrix $\gamma_{A}$. In addition, we shall define a matrix element of an arbitrary operator Ô evaluated with respect to two density matrices as

$$
\left\langle\gamma_{A}|\hat{\mathrm{O}}| \gamma_{B}\right\rangle=\iint \gamma_{A}\left(r, r^{\prime}\right) \hat{\mathrm{O}} \gamma_{B}\left(r, r^{\prime}\right) \mathrm{d} r \mathrm{~d} r^{\prime} .
$$

In particular, we will encounter matrix elements of the electron repulsion operator, which are given by

$$
\left\langle\gamma_{A}\left|r_{12}^{-1}\right| \gamma_{B}\right\rangle=\sum_{p q} \gamma_{p q}^{A} \sum_{r s} \gamma_{r s}^{B}(p r \mid q s)
$$

In the case of the 1TDM, we use the additional shorthand notation

$$
\langle\hat{\mathrm{O}}\rangle_{\mathrm{ex}}=\frac{\left\langle\gamma_{0 I}|\hat{\mathrm{O}}| \gamma_{0 I}\right\rangle}{\Omega}
$$

which is interpreted as an expectation value of the effective exciton wavefunction $\gamma_{0 I} \cdot \frac{37}{3}$ Here, the denominator is the squared norm of the exciton wavefunction $\Omega$, evaluated as

$$
\Omega=\left\langle\gamma_{0 I} \mid \gamma_{0 I}\right\rangle=\operatorname{tr}\left(\gamma_{0 I} \gamma_{0 I}^{\mathrm{T}}\right)=\sum_{p q}\left|\gamma_{p q}^{O I}\right|^{2}
$$

Below, we will also encounter expressions of the form $\iint \rho_{A}\left(r_{1}\right) r_{12}^{-1} \rho_{B}\left(r_{2}\right) \mathrm{d} r_{1} \mathrm{~d} r_{2}$ representing the electrostatic repulsion between the charge distributions $\rho_{A}$ and $\rho_{B}$, which cannot be directly written using the above notation. Therefore, we rearrange these terms in the following form

$$
\begin{array}{r}
\iint \frac{\rho_{A}\left(r_{1}\right) \rho_{B}\left(r_{2}\right)}{r_{12}} \mathrm{~d} r_{1} \mathrm{~d} r_{2}=\int \rho_{A}\left(r_{1}\right)\left(\int \frac{\rho_{B}\left(r_{2}\right)}{r_{12}} d r_{2}\right) d r_{1}= \\
\int \rho_{A}\left(r_{1}\right) \hat{\mathrm{V}} \rho_{B}\left(r_{1}\right) d r_{1}=\left\langle\rho_{A}|\hat{\mathrm{V}}| \rho_{B}\right\rangle .
\end{array}
$$

where $\hat{\mathrm{V}} \rho_{B}\left(r_{1}\right)$ is the electrostatic potential (ESP) induced by $\rho_{B} \cdot{ }^{93}$ Within this interpretation, the two-body electron-repulsion integral can be visualised as an overlap between the density of $\rho_{A}$ and the ESP induced by $\rho_{B}$, or vice versa.

\subsection{Derivation and representation of the CIS energy}

In view of the discussion to follow, we present a self-contained derivation of the CIS excitation energies, here, and spend some effort into recasting the results into a memorable and intuitive form. The main conclusions and their physical implications will be laid out in Sec. 2.3. Within CIS, the excited-state wavefunction of state $I$ is given as

$$
\left|\Psi_{I}\right\rangle=\sum_{i a} C_{i a} \hat{\mathrm{a}}_{a}^{\dagger} \hat{\mathrm{a}}_{i}\left|\Phi_{0}\right\rangle
$$

where $\left|\Phi_{0}\right\rangle$ is the ground state Slater determinant, $\hat{\mathrm{a}}_{a}^{\dagger}$ and $\hat{\mathrm{a}}_{i}$ are the creation and annihilation operators, and $C_{i a}$ is the CIcoefficient. To keep the notation simple, we restrict the CIcoefficients and orbitals to be real. We use the convention that indices $a, b$ correspond to the excited electron, $i, j$ to the excitation hole, $k, l$ to any occupied orbitals, and that $p, q, r, s$ are arbitrary orbital indices.

Within CIS, the ground state density matrix and the 1TDM are given as

$$
\begin{aligned}
\gamma_{0}\left(r, r^{\prime}\right) & =\sum_{k}^{\mathrm{occ}} \phi_{k}(r) \phi_{k}\left(r^{\prime}\right) \\
\gamma_{0 I}\left(r_{h}, r_{e}\right) & =\sum_{i}^{\mathrm{occ} \text { virt }} \sum_{a} C_{i a} \phi_{i}\left(r_{h}\right) \phi_{a}\left(r_{e}\right) .
\end{aligned}
$$

The density matrices for the hole and electron are given as

$$
\gamma_{i j}^{h}=\sum_{a}^{\text {virt }} C_{i a} C_{j a}
$$

$$
\gamma_{a b}^{e}=\sum_{i}^{\mathrm{occ}} C_{i a} C_{i b}
$$

In addition, we will encounter the Fock matrix, whose matrix elements are defined ${ }^{89}$ in a spin-orbital basis of Hartree-Fock MOs as

$$
F_{p q}=h_{p q}+\sum_{k}^{\mathrm{occ}}[(p q \mid k k)-(p k \mid k q)]
$$

For canonical MOs, the Fock matrix is diagonal with elements

$$
F_{p q}=\varepsilon_{p} \delta_{p q}
$$

where $\varepsilon_{p}$ is the energy of the MO $\phi_{p}$.

Using these basic expressions we now proceed by evaluating 


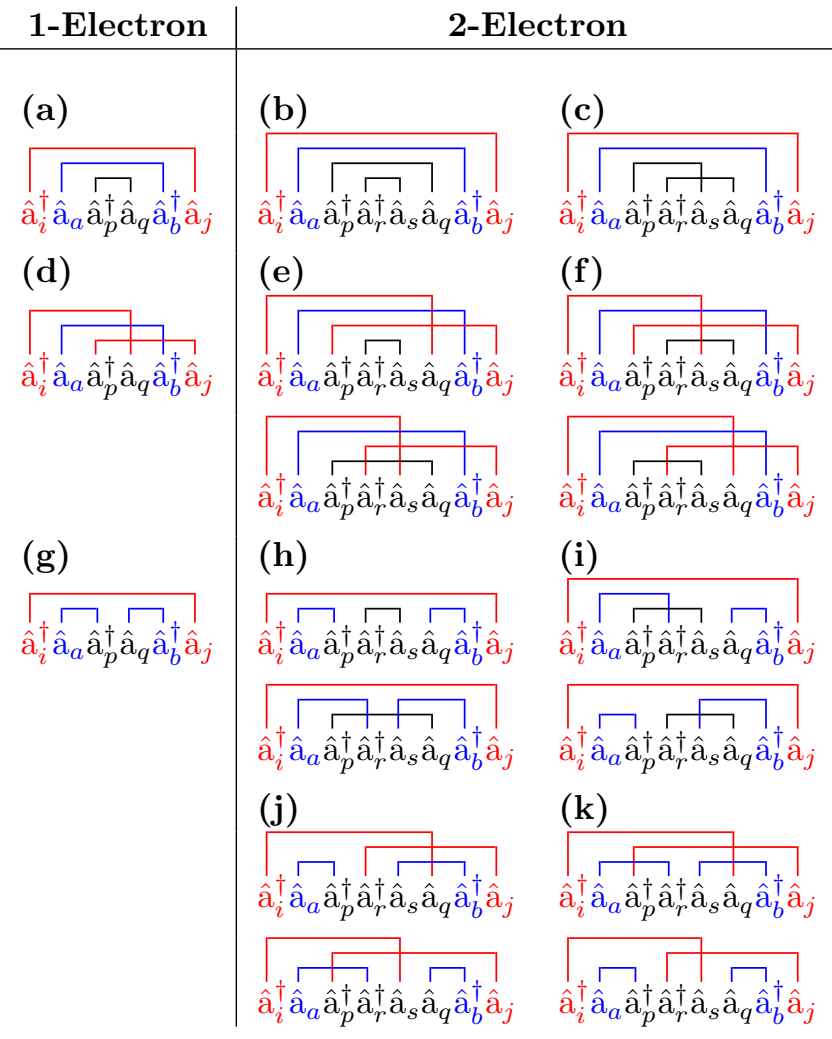

Fig. 2 Non-vanishing contractions of creation and annihilation operators giving rise to the CIS energy. The excitation hole and excited electron are marked in red and blue, respectively. The rows show the contributions of $(a-c)$ the ground state, (d-f) the excitation hole, $(g-i)$ the excited electron, and $(j-k)$ the dynamical interactions between hole and electron.

the excitation energy by inserting Eqs (2) and (24) into Eq. (1).

$$
\begin{aligned}
E_{I}=\sum_{i a j b} C_{i a} C_{j b} \sum_{p q} h_{p q}\left\langle\Phi_{0}\left|\hat{\mathrm{a}}_{i}^{\dagger} \hat{\mathrm{a}}_{a} \hat{\mathrm{a}}_{p}^{\dagger} \hat{\mathrm{a}}_{q} \hat{\mathrm{a}}_{b}^{\dagger} \hat{\mathrm{a}}_{j}\right| \Phi_{0}\right\rangle+ \\
\sum_{i a j b} C_{i a} C_{j b} \sum_{p q r s}(p q \mid r s)\left\langle\Phi_{0}\left|\hat{\mathrm{a}}_{i}^{\dagger} \hat{\mathrm{a}}_{a} \hat{\mathrm{a}}_{p}^{\dagger} \hat{\mathrm{a}}_{\mathrm{a}}^{\dagger} \hat{\mathrm{a}}_{s} \hat{\mathrm{a}}_{q} \hat{\mathrm{a}}_{\mathrm{a}}^{\dagger} \hat{\mathrm{a}}_{j}\right| \Phi_{0}\right\rangle
\end{aligned}
$$

At a first glance this equation looks quite daunting containing up to eightfold summations. However, by applying creation/annihilation operator algebra and re-expressing the results in terms of density matrices the equation can be brought into a memorable and physically intuitive form. When viewing Eq. (31), we first realise that it is not possible to obtain a non-vanishing result unless all the creation and annihilation operators are paired considering that they have to map the ground state determinant onto itself. This observation can be formalised via Wick's theorem ${ }^{86}$ and the task of pairing up the operators can be understood as finding the non-zero contractions between them. 88 These contractions are given in Fig. 2 2 showing that there are three 1-electron terms and fourteen 2-electron terms to consider.

Fig. 2 shows the non-vanishing terms in a compact fashion but it is hard to argue that this representation is much more intuitive than the initial equation (39). Therefore, we suggest proceeding via a diagrammatic ${ }^{88}$ formalism. To do so, we first define symbols for the four components of Eq. (31) as shown in Fig. 3 , the bra state (a), the ket state (b), the one-electron (c) and two-electron (d) parts of the Hamiltonian. In Fig. 3 (d), the left node corresponds to the first electron and the right node to the second, and if the electrons are written in charge-cloud notation according to Eq. (3), then we can directly insert the indices from the two nodes into the two sides of the two-electron integral. Having defined the diagrammatic representation, the task of finding all non-zero contractions corresponds to connecting all the arrows of the building blocks in Fig. 3. From these diagrams, we will subsequently be able to evaluate Eq. (39) by multiplying the relevant coefficients and matrix elements before summing over all the indices. In addition, the sign of the term is determined by the question whether the sum of the number of hole lines and loops is an even or odd number. 88

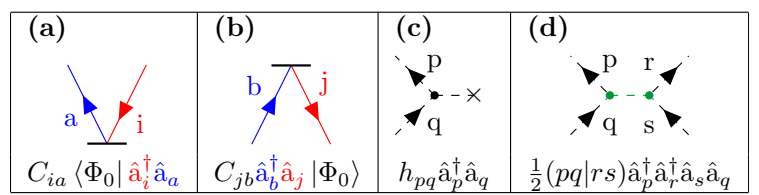

Fig. 3 Building blocks used to represent Eq. (31) in a diagrammatic form; (a) the bra state, (b) ket state, (c) the one-electron and (d) the twoelectron contributions. The excitation hole is marked in red, the excited electron in blue, and the Coulomb interaction in green.

In Fig. 2 (a-c), we have generally matched the $\hat{\mathrm{a}}_{i}^{\dagger}$ and $\hat{\mathrm{a}}_{j}$ as well as the $\hat{\mathrm{a}}_{a}$ and $\hat{\mathrm{a}}_{b}^{\dagger}$ indices. The analogous diagrams are constructed in Fig. 4 (a-c) by connecting the bra and ket building blocks given in Fig. 3 (a) and (b) via the red and blue lines. This generally leads to a term $\sum_{i a}\left|C_{i a}\right|^{2}=1$, which cancels out for normalised wavefunctions. Thus, in Fig. 4 (a) only the one-electron Hamiltonian remains and we are left with the term

$$
\sum_{i a}\left|C_{i a}\right|^{2} \sum_{k}^{\mathrm{occ}} h_{k k}=\operatorname{tr}\left(\gamma_{0} \mathbf{h}\right)
$$

which we have rewritten as the trace of the ground-state density matrix $\gamma_{0}$ with the one-electron Hamiltonian $\mathbf{h}$ according to Eqs (18) and (25). The next term (b) is evaluated as

$$
\frac{1}{2} \sum_{k l}(k k \mid l l)=\frac{1}{2}\left\langle\rho_{0}|\hat{\mathrm{V}}| \rho_{0}\right\rangle
$$

where we have used Eq (23). The ground state density $\rho_{0}$ enters according to Eq. (25) considering that the indices $k$ and $l$ are both only on their respective side. There are three loops and three hole lines $(i, k, l)$, which adds up to an even number meaning 88 that the term enters with a positive sign. Term (b) has a clear physical interpretation - it is the electrostatic repulsion of the ground state density with respect to itself, the so-called Hartree term. In line with Eq. (23), we visualise it by plotting the density along with the ESP realising that the value of $\left\langle\rho_{0}|\hat{\mathrm{V}}| \rho_{0}\right\rangle$ is given as the overlap between the two charge distributions. ${ }^{94} \mathrm{Fig} .4$ (c) is an exchange contribution given as

$$
-\frac{1}{2} \sum_{k l}(k l \mid l k)=-\frac{1}{2}\left\langle\gamma_{0}\left|r_{12}^{-1}\right| \gamma_{0}\right\rangle
$$

This term possesses a negative sign considering that the diagram 
contains two loops and three hole lines. Unlike term (b), this term has no intuitive interpretation. It is a non-local interaction involving the density matrices. Nonetheless, we can appreciate that the terms (b) and (c) both contain integrals of the form $(k k \mid k k)$ with opposite signs and that these are therefore cancelled between them. These terms would require a spin-orbital $\phi_{k}$ to be doubly occupied and are thus in violation of the Pauli exclusion principle. Thus, we can see that a major role of the exchange term (c) is the cancellation of the exclusion-principle violating (EPV) terms. ${ }^{88}$ In other words, the non-local exchange term in Hartree-Fock theory naturally cancels out the self-interaction error. Conversely, it is one of the major challenges in density functional theory to do so with only local information. 95

The combination of terms (a-c) yields the Hartree-Fock ground state energy

$$
E_{0}=\operatorname{tr}\left(\gamma_{0} \mathbf{h}\right)+\frac{1}{2}\left\langle\rho_{0}|\hat{\mathrm{V}}| \rho_{0}\right\rangle-\frac{1}{2}\left\langle\gamma_{0}\left|r_{12}^{-1}\right| \gamma_{0}\right\rangle .
$$

To summarise, the ground state energy is composed of a oneelectron term representing the kinetic energy and the electronnuclear attraction, the Hartree term, which is the self-repulsion of the density, and a non-local exchange term.

Next, we consider the terms (d-f). These are obtained by contracting the $\hat{\mathrm{a}}_{a}$ and $\hat{\mathrm{a}}_{b}^{\dagger}$ operators in Fig. 2 (d-f) and, accordingly, by connecting the electron lines (blue) of the bra and ket states in Fig. 4 (d-f). All five possible terms are shown in Fig. 2 (df) while only the three diagrams that are unique with respect to an interchange of the electrons are shown in in Fig. 4 (d-f). In the diagrams, we see that the lines pertaining to the excitation hole (red) interact with the operator, and algebraically this is represented by the term $\sum_{a} C_{i a} C_{j a}$, which we identify with the hole density matrix $\gamma_{h}$ according to Eq. (27). Furthermore, we find that the second row in Fig. 4 has exactly the same form as the first row with the exception of a replacement $\gamma_{0} \rightarrow \gamma_{h}$ or $\rho_{0} \rightarrow \rho_{h}$. These terms are interpreted as the one-electron energy of the hole (d), its Coulomb repulsion with respect to the ground state density (e), and its exchange energy with the ground state (f). Note that these terms have opposite signs with respect to Fig. 4 (a-c), i.e. it is energetically favourable if the hole has a large one-electron energy and strong repulsion with the ground state. The Coulomb attraction between $\rho_{\mathrm{h}}$ and $\rho_{0}$ is represented in Fig. 4 (e) via the hole density and the ground-state ESP. It is favourable to form the hole in an area of negative (blue) rather than positive (red) ESP. Panel (f) represents a non-local exchange term that cannot be immediately put into a pictorial representation. However, it can be appreciated that the hole is generally part of the ground-state density. Therefore, we can assume that the dominant term is the self-interaction of the hole density $\left\langle\rho_{h}|\hat{\mathrm{V}}| \rho_{h}\right\rangle$ unless non-trivial correlation effects play a role and we use this term in the pictorial representation of Fig. 4 ( $f$ ). In combination, the three terms in (d-f) reflect the definition of the Fock matrix in Eq. 29) and we can rewrite them in more compact form as $-\operatorname{tr}\left(\gamma_{h} \mathbf{F}\right)$, which according to Eq. (30) is also equivalent to a weighted sum over orbital energies $-\sum_{i a}\left|C_{i a}\right|^{2} \varepsilon_{i}$.

The energy of the excited electron is shown in Fig. 4 (g-i). There is a close analogy to the terms related to the hole with the exception that the signs are reversed. The combination of the terms (g-i) yields, again, a weighted sum of the orbital energies $\varepsilon_{i}$ or equivalently a contraction of the Fock matrix $F_{i j}$ with the electron density matrix. However, the crucial difference between the hole and the electron is that that the electron interacts with the full ground-state density as shown in Fig. 4(h) whereas the hole only interacts with $n-1$ electrons considering that its self-interaction is cancelled in term (f). Consequently, HOMO and LUMO energies in Hartree-Fock theory are estimates of the ionisation potential (IP) and electron affinity (EA) according to Koopmans' theorem. The HOMO-LUMO gap is a good guess for the fundamental gap of the system but it is not a good guess of the excitation energy ${ }^{96}$ due to the importance of the electronhole Coulomb attraction, i.e. term (k) to be discussed below. Finally, we note that terms ( $g$-i) are analogous to the first order diagrammatic representation of the electron propagator within Hartree-Fock theory (sometimes denoted the cross, tadpole and oyster). .97

In the final row of Fig. 4, marking the interactions between electron and hole, there are no one-electron terms but two twoelectron terms are present deriving from the Coulomb (j) and exchange (k) potential of Hartree-Fock theory. Interestingly term (j) has exchange-like properties while (k) is seen as a Coulomb interaction, ${ }^{96}$ as explored in more detail below. It should also be noted that the first-order diagrammatic description of the polarisation propagator 97 is composed of two diagrams that are analogous to terms ( $\mathrm{j}$ ) and $(\mathrm{k})$ illustrating the equivalence of $\operatorname{ADC}(1)$ for the polarisation propagator and CIS.

Term (j), given as $\left\langle\rho_{0 I}|\hat{\mathrm{V}}| \rho_{0 I}\right\rangle$, is a repulsive term and can be represented graphically as the repulsion of the transition density with respect to itself. This term vanishes for triplet states while it generally raises the energy of singlet states. The final term (k) derives from the Hartree-Fock exchange potential and can be identified with the Coulomb binding energy of the correlated electronhole pair. $\frac{54 \mid 98}{5}$ The term is given as $\left\langle\gamma_{0 I}\left|r_{12}^{-1}\right| \gamma_{0 I}\right\rangle$ and assuming that the wavefunction is normalised, we can rewrite it according to Eq. 21) as an expectation value of the exciton wavefunction with respect to the inverse electron hole distance $\left\langle r_{12}^{-1}\right\rangle_{\mathrm{ex}}$. It is, thus, closely related to two of our previously defined descriptors, the linear and root-mean-square electron-hole separations ${ }^{99}$

$$
\begin{aligned}
& d_{h \rightarrow e}=\left\langle r_{12}\right\rangle_{\mathrm{ex}} \\
& d_{e x c}=\sqrt{\left\langle r_{12}^{2}\right\rangle_{\mathrm{ex}}},
\end{aligned}
$$

and we will use those quantities to estimate the magnitude of the Coulomb interaction. In addition, we have previously defined a related dimensionless quantity, the electron-hole correlation coefficient $R_{h e}{ }^{.99} R_{h e}$ takes values between -1 and 1 , where a positive value means that the electron and hole dynamically attract each other while a negative value means dynamical avoidance. In the present context, we can understand this value in the sense that in the case of a positive $R_{h e}$ value there is a dynamic enhancement of the Coulomb attraction but also of exchange repulsion. In large molecules, we can find positive correlation coefficients well above $0.5^{54 / 70}$ indicating that long-range Coulomb attrac- 


\begin{tabular}{|c|c|c|c|}
\hline 1-Electron & Coulomb & Exchange & Combined \\
\hline $\begin{array}{l}\text { (a) } \\
\sum_{i a}\left|C_{i a}\right|^{2} \sum_{k} h_{k k} \\
=\operatorname{tr}\left(\gamma_{0} \mathbf{h}\right)\end{array}$ & (b) & $\begin{array}{c}\text { (c) } \\
-\frac{1}{2} \sum_{k l}(k l \mid l k)=-\frac{1}{2}\left\langle\gamma_{0}\left|r_{12}^{-1}\right| \gamma_{0}\right\rangle \\
\approx-\frac{1}{2} \sum_{k}(k k \mid k k)\end{array}$ & $E_{0}$ \\
\hline $\begin{array}{l}\text { (d) } \\
-\sum_{a i j} C_{i a} C_{j a} h_{j i}\end{array}$ & $\begin{array}{l}\text { (e) } \\
-\sum_{a i j k} C_{i a} C_{j a}(j i \mid k k)=-\left\langle\rho_{h}|\hat{\mathrm{V}}| \rho_{0}\right\rangle\end{array}$ & $\begin{array}{l}\text { (f) } \\
\sum_{a i j k} C_{i a} C_{j a}(j k \mid k i)=\left\langle\gamma_{\mathrm{h}}\left|r_{12}^{-1}\right| \gamma_{0}\right\rangle\end{array}$ & $\begin{array}{c}-\operatorname{tr}\left(\gamma_{h} \mathbf{F}\right) \\
=-\sum_{i a}\left|C_{i a}\right|^{2} \epsilon_{i}\end{array}$ \\
\hline $\begin{array}{l}\text { (g) } \\
\sum_{a b i}^{\mathrm{k}} C_{i a} C_{i b} h_{a b} \\
=\operatorname{tr}\left(\gamma_{e} \mathbf{h}\right)\end{array}$ & $\sum_{a b i k} C_{i a} C_{i b}(a b \mid k k)=\left\langle\rho_{e}|\hat{\mathrm{V}}| \rho_{0}\right\rangle$ & $-\sum_{a b i k} C_{i a} C_{i b}(k b \mid a k)=-\left\langle\gamma_{\mathrm{e}}\left|r_{12}^{-1}\right| \gamma_{0}\right\rangle$ & $\begin{aligned} & \operatorname{tr}\left(\gamma_{e} \mathbf{F}\right) \\
= & \sum_{i a}\left|C_{i a}\right|^{2} \epsilon_{a}\end{aligned}$ \\
\hline & $\begin{array}{l}\text { (j) } \\
\sum_{a b i j} C_{i a} C_{j b}(j b \mid a i)=\left\langle\rho_{0 I}|\hat{\mathrm{V}}| \rho_{0 I}\right\rangle\end{array}$ & $\begin{array}{l}\text { (k) } \\
-\sum_{a b i j} C_{i a} C_{j b}(j i \mid a b)=-\left\langle\gamma_{0 I}\left|r_{12}^{-1}\right| \gamma_{0 I}\right\rangle \\
\approx 600-C O S\end{array}$ & \\
\hline
\end{tabular}

Fig. 4 Diagrammatic representation of the CIS excitation energies. The columns show the one-electron, Coulomb and exchange contributions as well as their combined interpretation. The rows show the contributions of (a-c) the ground state, (d-f) the excitation hole, ( $g-i)$ the excited electron, and $(\mathrm{j}-\mathrm{k})$ the dynamical interactions between hole and electron. Each individual panel features diagrammatic and algebraic representations. Here, $i$ and $j$ refer to the excitation hole, $a$ and $b$ to the excited electron, and $k$ and $l$ to arbitrary doubly occupied orbitals in the ground state. If possible, a pictorial representation is given of a Coulomb interaction between two partial densities where the right-hand density is represented via its ESP $(\hat{V})$. Intense colours mean a large absolute value of the ESP while white represents a vanishing ESP. Only approximate pictorial representations can be given for the exchange terms and it is discussed in the main text under which conditions they are valid.

tion dominates whereas in small molecules some slightly negative $R_{h e}$ values are encountered $\sqrt{70 / 80}$ if exchange repulsion dominates. It is also worth pointing out that the correlation coefficient $R_{h e}$ has to vanish if $\mathrm{PR}_{\mathrm{NTO}}$ is exactly $1^{99}$ highlighting the connection between spatial correlation and multiconfigurational character.

In light of the discussion to follow, we also want to briefly sketch how to extend the CIS model to include de-excitations via the time-dependent Hartree-Fock (TDHF) method.96 Within TDHF the CI vector $C_{i a}$ is replaced by the excitation $\left(X_{i a}\right)$ and deexcitation $\left(Y_{i a}\right)$ vectors. On the diagonal blocks, excitations and de-excitations experience the same contributions already shown in Fig. 4. Two additional two-body terms come into play for coupling excitations and de-excitations. A possible diagrammatic representation of these terms, achieved by placing the $X_{i a}$ and $Y_{i a}$ both on the formal ket side of the diagram, is presented in Fig. 5 We find that the first term, shown in Fig.5 (a) possesses the same structure as the transition density repulsion [Fig. 4 (j)] with the exception that the $C_{i a}$ and $C_{j b}$ terms are replaced by $X_{i a}$ and $Y_{j b}$. However, the second term (b) does not resemble Fig. 4 (k) but it is a new type of interaction where the excitation hole is connected with the de-excited electron and vice versa. This term originates from non-local exchange 96 and occurs within TDDFT 


\begin{tabular}{|c|c|}
\hline Coulomb & Exchange \\
\hline (a) $X_{i a} Y_{j b}$ & (b) $X_{i a} Y_{j b}$ \\
\hline & \\
$\sum_{a b i j} X_{i a} Y_{j b}(a i \mid b j)$ & $-\sum_{a b i j} X_{i a} Y_{j b}(b i \mid a j)$ \\
\hline
\end{tabular}

Fig. 5 Diagrammatic representation of the coupling of the excitation $\left(X_{i a}\right)$ and de-excitation $\left(Y_{j b}\right)$ amplitudes in time-dependent Hartree-Fock theory and hybrid TDDFT.

only through hybrid functionals.

Given four orbital indices $a, b, i, j$, it is generally possible to construct three different two-electron integrals as we have three choices to pair any given index with another one. The first option, pairing the indices belonging to the bra and ket states, yields the transition-density-type integral $(a i \mid b j)$ found for the diagonal terms [Fig. 4(j)] as well as for the coupling [Fig. [5 (a)]. The second option, pairing the holes and electrons, gives a Coulomb integral $(i j \mid a b)$ found only in the diagonal terms [Fig. $4(\mathrm{k})$ ]. The third option gives a mixed transition-density-type integral (bi $\mid a j)$ only contributing to the coupling. In summary, we conclude that the coupling between excitations and de-excitations is mediated via transition-density-type integrals and we can assume that there is a relation between the transition density selfrepulsion $\left\langle\rho_{0 I}|\hat{\mathrm{V}}| \rho_{0 I}\right\rangle$ and the coupling between excitations and de-excitations.

\subsection{The energy of a correlated electron-hole pair}

Having derived the individual terms contributing to the CIS energy, we can now proceed to use this information for a physical interpretation. First, we can combine the three terms on the right column of Fig. 4 with the terms (j) and (k) to write the CIS excitation energy $\left(\Delta E=E_{I}-E_{0}\right)$ in close analogy to the Hartree-Fock ground state energy [Eq. (35)] as

$$
\Delta E=\operatorname{tr}\left(\left(\gamma_{e}-\gamma_{h}\right) \mathbf{F}\right)+\left\langle\rho_{0 I}|\hat{\mathrm{V}}| \rho_{0 I}\right\rangle-\left\langle\gamma_{0 I}\left|r_{12}^{-1}\right| \gamma_{0 I}\right\rangle .
$$

where $\gamma_{e}-\gamma_{h}$ is the (unrelaxed) CIS difference density matrix. Alternatively, the MO energies can be inserted

$$
\Delta E=\sum_{i a}\left|C_{i a}\right|^{2}\left(\varepsilon_{a}-\varepsilon_{i}\right)+\left\langle\rho_{0 I}|\hat{\mathrm{V}}| \rho_{0 I}\right\rangle-\left\langle\gamma_{0 I}\left|r_{12}^{-1}\right| \gamma_{0 I}\right\rangle .
$$

Thus, the final result is a sum of MO energies and two contributions going beyond that, which can be seen as excited-state versions of the Hartree and exchange energies. 100

It is worth pointing out here that $\gamma_{h}, \gamma_{e}$, and $\rho_{0 I}$ are all directly computed from the 1TDM $\gamma_{0 I}$. Thus, the 1TDM can be seen as the fundamental quantity determining the energy of the correlated electron-hole pair. ${ }^{101}$ By contrast, the difference density matrix only covers the first term in Eq. (38) rigorously and gives no indication of exchange repulsion or any dynamic effects related to Coulomb attraction. From this discussion it also follows that two states reached via the same orbital transitions and, thus, also possessing the same excited-state densities may well differ in their energies as long as at least one of the terms $\left\langle\rho_{0 I}|\hat{\mathrm{V}}| \rho_{0 I}\right\rangle$ or $\left\langle\gamma_{0 I}\left|r_{12}^{-1}\right| \gamma_{0 I}\right\rangle$ is different among the states. In these cases the relative signs of the different transitions carry the crucial information as is the case, e.g., for the difference between singlet and triplet states, between excitonic and charge-resonance states, $\frac{76] 102}{102}$ and between the ionic and covalent states of alternant hydrocarbons. $.38,40 / 42$

Both of the extra terms in Eq. (38) have clear physical interpretations and important consequences on observed molecular properties. The first term $\left\langle\rho_{0 I}|\hat{\mathrm{V}}| \rho_{0 I}\right\rangle$ is the primary reason for the "exchange splitting" between singlet and triplet excited states and the main handle one has for tuning their relative energies. It is generally large if there is a large spatial overlap between the involved orbitals and it becomes small if the orbitals are separated in space or if their overlap is minimised through a twisted molecular geometry. ${ }^{222}$ It is affected by the interference between different orbital excitations, and we shall explore below that this term is also affected by the involvement of $\sigma$-orbitals. The transition density repulsion is also at the foundation of Förster coupling, 103 and the energy of plasmonic states depends strongly on this term. .81104

It is interesting to note that the exchange splitting has a relation to the transition moment defined in Eq. (14) considering that both quantities depend on the transition density. It immediately follows that both terms vanish if the transition density vanishes, as is the case for triplet states and CT states between chromophores that are completely separated in space. More generally, there is a correlation between these two terms as a charge distribution with a strong dipole moment tends to also induce a larger electrostatic potential. This leads to the general rule that there is an energetic penalty for bright states, which holds in the cases of singlet vs triplet states, $\pi \pi^{*}$ vs $n \pi^{*}$ states, and locally excited vs CT states, as we shall explore in some more detail below.

The final term $\left\langle\gamma_{0 I}\left|r_{12}^{-1}\right| \gamma_{0 I}\right\rangle$ is interpreted as the Coulomb binding energy of the electron-hole pair $\frac{54 \mid 98}{5}$ and is, in general, a nontrivial correlated term. It is responsible for the difference between the energies of Frenkel excitons and charge resonance states in dimers ${ }^{76 / 102}$ as well as for the energies of different exciton bands in conjugated polymers. ${ }^{34}$ The underlying correlations can be visualised with the help of pseudocolor plots of the $1 \mathrm{TDM}^{\mathbf{7 4} 76}$ or via conditional densities. 42

Finally, we shall compare the energy of the electron-hole pair as derived above with the related expressions obtained by TDDFT and GW/BSE. Both, the TDDFT and GW/BSE methods include the $\left\langle\rho_{0 I}|\hat{\mathrm{V}}| \rho_{0 I}\right\rangle$ term in unaltered form. ${ }^{85|96| 105}$ By contrast, the $\left\langle\gamma_{0 I}\left|r_{12}^{-1}\right| \gamma_{0 I}\right\rangle$ term is not included at all in typical linear-response TDDFT computations with approximate local functionals. The absence of this term is at the heart of the infamous chargetransfer problem of TDDFT ${ }^{52}$ and its related problems for large molecules ${ }^{43 / 44}$ and, specifically, conjugated polymers. ${ }^{[5354}$ To overcome this problem, it is common practice to include HartreeFock exchange in the computation via a hybrid functional. However, it is well-established that good results are generally not obtained by including the full amount of "exact exchange" but only a fraction $c_{H F}$ of it. Thus, the Coulomb interaction is replaced by 
the term

$$
-c_{H F}\left\langle\gamma_{0 I}\left|\frac{1}{r_{12}}\right| \gamma_{0 I}\right\rangle=-\left\langle\gamma_{0 I}\left|\frac{1}{\varepsilon} \frac{1}{r_{12}}\right| \gamma_{0 I}\right\rangle .
$$

On the left-hand-side the multiplication by $c_{H F}$ is shown explicitly while on the right-hand-side, this is interpreted as an effective dielectric constant of magnitude $\varepsilon=1 / c_{H F}$ uniformly screening the Coulomb interaction within the molecule. Improved results are obtained by attenuating the Coulomb interaction in a non-uniform way ${ }^{106}$ within a range separated functional. For example, the CAM-B3LYP functional, 106 approximates the electronhole interaction as

$$
-\left\langle\gamma_{0 I}\left|\frac{\alpha+\beta \operatorname{erf}\left(\mu r_{12}\right)}{r_{12}}\right| \gamma_{0 I}\right\rangle
$$

where $\alpha, \beta$, and $\mu$ are parameters. In the GW/BSE method, a somewhat different approach is used and the electron-hole interaction is described by the name-giving screened Coulomb interaction $W .{ }^{84}$ Various schemes for constructing $W$ have been suggested, e.g. the plasmon-pole $\frac{107}{10}$ or random phase approximations. 108 Conversely, wavefunction based methods have to incorporate the same physics of a screened electron-hole interaction via orbital relaxation $\sqrt{55 \mid 109}$ or dynamic correlation effects.

\subsection{Simplifications for a two-orbital model}

The equations shown can be simplified in the case that only two spatial orbital are involved, i.e. in the case of a two-orbital twoelectron model (TOTEM) $\stackrel{100}{\square}$ This two-orbital case may be immediately apparent when looking at the excitation vector in terms of canonical orbitals, e.g. for a plain HOMO-LUMO transition. But, we want to use the more general condition here that the 1TDM can be decomposed into one single pair of NTOs $\psi^{h} / \psi^{e}$ according to Eq. (8). Using these orbitals, the spin-adapted singlet and triplet states are constructed according to

$$
\left|\Psi_{S / T}\right\rangle=\frac{1}{\sqrt{2}}\left(\hat{\mathrm{a}}_{e \alpha}^{\dagger} \hat{\mathrm{a}}_{h \alpha} \pm \hat{\mathrm{a}}_{e \beta}^{\dagger} \hat{\mathrm{a}}_{h \beta}\right)\left|\Phi_{0}\right\rangle
$$

where the + refers to singlet and the - to triplet. $\hat{a}_{e \alpha}^{\dagger}$ and $\hat{a}_{h \alpha}$ are the creation and annihilation operators referring to the two NTOs and acting on the respective spin-orbital. The equations for the singlet and triplet excitation energies are readily constructed as 100

$$
\begin{aligned}
\Delta E_{S} & =\varepsilon_{e}-\varepsilon_{h}+2(h e \mid e h)-(h h \mid e e) \\
& =\varepsilon_{e}-\varepsilon_{h}+\left\langle\rho_{0 I}|\hat{\mathrm{V}}| \rho_{0 I}\right\rangle-\left\langle\rho_{h}|\hat{\mathrm{V}}| \rho_{e}\right\rangle \\
\Delta E_{T} & =\varepsilon_{e}-\varepsilon_{h}-(h h \mid e e)=\varepsilon_{e}-\varepsilon_{h}-\left\langle\rho_{h}|\hat{\mathrm{V}}| \rho_{e}\right\rangle .
\end{aligned}
$$

Thus, in the case of a two-orbital model, the correlated interaction term $\left\langle\gamma_{0 I}\left|r_{12}^{-1}\right| \gamma_{0 I}\right\rangle$ is replaced by the static Coulomb attraction $\left\langle\rho_{h}|\hat{\mathrm{V}}| \rho_{e}\right\rangle$ between the electron and hole densities. This approximation is indicated pictorially in Fig. 4 (k).

It is interesting to discuss Eqs (43) and (44) in the context of charge transfer states, i.e. states where the hole and electron are separated in space. First, if the spatial overlap between $\psi^{h}$ and $\psi^{e}$ is small, it follows that $\rho_{0 I}$ becomes small everywhere in space and as a consequence the term $\left\langle\rho_{0 I}|\hat{\mathrm{V}}| \rho_{0 I}\right\rangle$ is small. As a consequence the singlet and triplet energies become approximately equal. Second, as discussed above, within Hartree-Fock, we can identify the orbital energies with the EA and IP of the system according to $\varepsilon_{e} \approx \mathrm{EA}$ and $-\varepsilon_{h} \approx \mathrm{IP}$. Third, for well-separated charge transfer states, we can approximate the hole and particle densities as point charges separated by a distance $d$, and their interaction becomes $\left\langle\rho_{h}|\hat{\mathrm{V}}| \rho_{e}\right\rangle \approx 1 / d$. Under these assumptions the excitation energies can be written as 96

$$
\Delta E_{S} \approx \Delta E_{T} \approx \mathrm{EA}+\mathrm{IP}-\frac{1}{d},
$$

i.e. the sum of electron affinity and ionisation potential minus a distance dependent exciton binding term. In practice, we can use the values $d_{h \rightarrow e}$ and $d_{e x c}$ as defined in Eqs (36) and (37) to estimate the distance $d .37$

Finally, a word on the performance of linear-response TDDFT is in order. If local functionals are used, the equations become even simpler because the (hh|ee) term disappears from Eqs (43) and (44), and is replaced by an alternative term deriving from the local exchange-correlation potential ${ }^{96}$ that is usually smaller. At first sight this may appear like a gross negligence introducing errors on the order of several $\mathrm{eV}$ in small molecules. However, it is the magic of TDDFT that a similar term is incorporated into the orbital energies, in particular through neglect of the $(h h \mid h h)$ term of Fig. 4 (f) and more generally, we can hope that the terms (f), (i), and (k) cancel out approximately. Thus, we are left with the situation that TDDFT with approximate local functionals when applied to two-orbital valence excitations of small molecules does not only provide good excitation energies but that already the orbital energy differences themselves are good approximations to the excitation energies. $\frac{96 / 110}{}$ Conversely, it has to be pointed out that within DFT the HOMO energy is an approximation to the IP only for the exact functional, 111 but is usually grossly underestimated with standard functionals, 110 and that within DFT the LUMO energy is never an approximation to the EA. It is, therefore, important to stress the difference between the meaning of orbital energies between Hartree-Fock and DFT. Within HartreeFock, the orbital energies approximate the IP and EA but their difference is usually a bad guess for the excitation energy. DFT orbital energies, on the other hand, are a good approximation for the optical gaps but should not be used to represent the IP and EA.

\subsection{Classification of excited states and validity of the models}

In the above sections, we have spent some effort in trying to present an intuitive and memorable model for the CIS excitation energies. However, the objective was not to understand the CIS model as such, but to provide general expressions determining the energy of a correlated electron-hole pair. In this section, we will therefore consider different descriptors applicable to highlevel quantum chemistry computations that indicate whether the applied model is valid and whether the simplified two-orbital model of Sec. 2.4 is even applicable. In addition, we will identify two phenomena beyond the CIS model: secondary orbital relax- 
ation and de-excitations. To this end, we use three previously defined descriptors, the single-excitation character $\Omega, \sqrt[6166]{60}$ the natural transition orbital participation ratio $\mathrm{PR}_{\mathrm{NTO}}, \frac{, 70,76 / 90}{\text { and the }}$ promotion number $p \cdot \frac{5961}{5 i n a l l y, ~ w e ~ i n t r o d u c e ~ a ~ n e w ~ d e s c r i p t o r, ~}$ the expectation value $P_{h e}$ of the electron-hole permutation operator. These four descriptors rely entirely on the eigenvalues and singular values of the density matrices and do not include any information about shape or the spatial distribution.

The single-excitation character $\Omega$ has already been defined in Eq. (22). The value of $\Omega$ is exactly 1 in the case of CIS and we can, thus, assume that the approximate CIS picture holds when $\Omega \approx 1$ in the sense that the excited state is generated as a linear combination of single excitations from the ground state. Singly excited states computed by correlated ab initio methods are typically described by $\Omega$ values above 0.8 and we can use this as a threshold for the validity of the model. In principle, it is also possible to obtain $\Omega$ values larger than 1 if a ground state with several open shells is considered ${ }^{112}$ but this seems to be only a curiosity.

To measure the multiconfigurational character of the excited state, we use the $\mathrm{PR}_{\mathrm{NTO}}$ value defined in Eq. (9), which gives the number of configurations involved in the excited state. Whereas, the $\Omega$ value specifies whether the CIS model is applicable at all, $\mathrm{PR}_{\mathrm{NTO}}$ specifies whether the simplified two-orbital model of Section 2.4 is applicable.

To quantify the number of electrons moved during the excitation process, we compute the promotion number $p$, which is defined as the spatial integral over the attachment or detachment density ${ }^{59] 61}$ In the case of CIS, the value of $p$ exactly 1 and for correlated calculations one usually obtains larger values. $55 \mid 61 / 109113$

Finally, we want to suggest a new descriptor denoted $P_{h e}$. It is known that the 1TDM in the case of CIS is nilpotent, ${ }^{90}$ i.e. that

$$
\gamma_{0 I} \gamma_{0 I}=0
$$

where the equation is to be interpreted as a matrix multiplication. We will, therefore, use the non-nilpotency of the 1TDM as an additional measure for the deviations from CIS. This is analogous to the widely used practice of considering the non-idempotency (or non-duodempotency) of the ground state density matrix to measure deviations from Hartree-Fock theory and, thus, unpaired electrons. $114 \mid 119$ To put the equation into a memorable form, we introduce the electron-hole permutation operator $\mathscr{P}_{h e}$, which by acting on the 1TDM exchanges the coordinates of the hole and electron, i.e.

$$
\mathscr{P}_{h e} \gamma_{0 I}\left(r_{h}, r_{e}\right)=\gamma_{0 I}\left(r_{e}, r_{h}\right)=\sum_{p q} \gamma_{p q}^{0 I} \phi_{p}\left(r_{e}\right) \phi_{q}\left(r_{h}\right)
$$

By inserting Eq. (47) into Eq. (21), we find that the expectation value of the electron-hole permutation operator is

$$
P_{h e}=\left\langle\mathscr{P}_{h e}\right\rangle_{\mathrm{ex}}=\frac{\operatorname{tr}\left(\gamma_{0 I} \gamma_{0 I}\right)}{\Omega},
$$

which naturally measures deviations from nilpotency as defined in Eq. (46). Note that Eq. (48) is closely related to the definition of $\Omega$ via Eq. (22). By application of the Cauchy-Schwarz inequal- ity, we find that

$$
-1 \leq P_{h e} \leq 1 .
$$

The value of $P_{h e}$ is zero for CIS while it may deviate for any wavefunction method that employs a correlated ground state. It is zero for TDDFT in the Tamm-Dancoff approximation. In case of full TDDFT, the response vector is divided into an excitation part $X_{i a}$ and a de-excitation part $Y_{i a}, 96$ and the 1TDM can be written as 120

$$
\gamma_{0 I}\left(r_{h}, r_{e}\right)=\sum_{i}^{\mathrm{occ}} \sum_{a}^{\mathrm{virt}}\left[X_{i a} \phi_{i}\left(r_{h}\right) \phi_{a}\left(r_{e}\right)+Y_{i a} \phi_{a}\left(r_{h}\right) \phi_{i}\left(r_{e}\right)\right]
$$

This 1TDM will produce a non-vanishing expectation value with respect to $\mathscr{P}_{h e}$ whenever there are excitations and de-excitations involving the same pair of orbitals and the value of $P_{h e}$ in the case of TDDFT is given as

$$
P_{h e}=\frac{\sum_{i a} X_{i a} Y_{i a}}{\|\mathbf{X}\|^{2}+\|\mathbf{Y}\|^{2}}
$$

We, therefore, propose $P_{h e}$ as a measure for the de-excitation character. Its value for TDDFT is given by Eq. (51) while it is welldefined for any method that produces a 1TDM. We shall explore below whether similar values are obtained for TDDFT and wavefunction based methods. To finish, we want to point out that a similar measure of particle-hole parity was considered in Ref. 121 only that the interpretation was somewhat different due to the explicit enforcement of particle-hole symmetry. We also want to point the reader to a discussion of de-excitations in Ref. 122

In summary, we can use the four descriptors $\Omega, \mathrm{PR}_{\mathrm{NTO}}, p$, and $P_{h e}$ to classify excited states as shown in Table 1 . If the $\Omega$ and $\mathrm{PR}_{\mathrm{NTO}}$ values are close to one, then we have a simple orbital-toorbital transition according to Eq. (42) and the simplified relations in Section 2.4 are valid. This category usually encompasses the $n \pi^{*}$ states of organic molecules. It also applies to a certain subset of $\pi \pi^{*}$ states, most notably the $L_{a}$ states 123 of aromatic systems and HOMO-LUMO transitions in push-pull aromatic systems. If $\Omega$ is still close to 1 but the $\mathrm{PR}_{\mathrm{NTO}}$ value is significantly larger than one, then the CIS picture still holds but it is generally not possible to understand a state in a two-orbital picture. In such a case non-trivial interference effects come into play, which can be interpreted in terms of electron correlation, 124 and it is necessary to use the full model of Eq. (39). Larger PR $_{\mathrm{NTO}}$ values are obtained for the $L_{b}$ states of aromatic molecules and for other states that are reached via quasi-degenerate orbital transitions. ${ }^{7080} \mathrm{PR}_{\mathrm{NTO}}$ values larger than one are also found for coupled chromophores ${ }^{\sqrt{76} 125}$ where the relative signs of the configurations allow to differentiate between excitonic and charge resonance states, $\frac{102}{102}$ as well as for conjugated polymers showing longrange excitonic correlation. $34|70| 99$ A third class of excited states are characterised by $\Omega$ values significantly below 1 , and these are identified as multiply excited states 61$] 99 \mid 109$ making the presented model invalid. A fourth class is observed for cases where the $\Omega$ value is still reasonably close to 1 but $p$ is significantly larger. These can still seen be as singly excited states only that secondary orbitals are rearranged in the process. 55|109|113 The model presented here should be valid only that the orbital relaxation could 
Table 1 Classification of excited states according to the single-excitation character $\Omega$, the NTO participation ratio $\mathrm{PR}_{\mathrm{NTO}}$, the promotion number $p$, and the expectation value of the electron-hole permutation operator $P_{h e}$.

\begin{tabular}{ll}
\hline Condition & Classification \\
\hline$\Omega \approx 1, \mathrm{PR}_{\mathrm{NTO}} \approx 1$ & Simple orbital-to-orbital transition \\
$\Omega \approx 1, \mathrm{PR}_{\mathrm{NTO}}>1.2$ & Correlated singly excited state \\
$\Omega<0.8$ & Multiply excited state \\
$\Omega \approx 1, p>1.2$ & State with orbital relaxation \\
$\left|P_{h e}\right|>0.2$ & State with de-excitations \\
\hline
\end{tabular}

be seen as a charge screening effect. Larger $p$ values are often observed for $n \pi^{*}$ states 109 and charge transfer states, particularly in transition metal complexes. ${ }^{[55}$ Methodologically speaking, states with larger $p$ values require additional flexibility in the wavefunction description but it might suffice to use an orbital-optimised version of the CIS method 126 rather than a truly dynamically correlated method. The final class of states, identified by nonvanishing $P_{h e}$ values, corresponds to states with significant deexcitation character. We do not have experience regarding this value but following Refs 5657, we can posit that this quantity is more important for triplet than for singlet states, and that it depends on non-local exchange.

\section{Computational Details}

The molecular geometries of uracil and the polyacenes were optimised at the PBEh-3c level of theory. 127 The geometry of octatetraene was taken from Ref. 81. Excited state computations on uracil were performed at the ADC(2) $82[128$ and TDDFT/PBE0 129 levels of theory both using the def2-SV(P) basis set ${ }^{130}$ as well as at the complete active space self-consistent field (CASSCF) level of theory placing 10 electrons in 7 active orbitals $(1 \times n, 4 \times \pi, 2 \times$ $\left.\pi^{*}\right)$, individually averaging over 3 singlet and 3 triplet states, and using the def2-SVP basis set. Computations on the polyacenes used the ADC(2) method in connection with the aug-cc-pVDZ basis set 131 following a previous study that highlighted the importance of diffuse basis functions in the description. ${ }^{113}$ The computation of conditional densities for anthracene was performed using only the def2-SV(P) basis set to avoid linear dependencies. Excited states of octatetraene were computed at the CIS/augcc-pVTZ level.131 ADC, TDDFT and CIS computations were performed with a developmental version of Q-Chem $5.2^{132}$ while CASSCF computations were done in OpenMolcas. 133|134 Wavefunction analysis, as described in detail in the previous section, was performed via the libwfa library ${ }^{135}$ using its interfaces to Q-Chem ${ }^{61}$ as well as OpenMolcas. ${ }^{113}$ Molecular geometries and input/output files of Q-Chem and OpenMolcas are provided via a separate repository. 136

The (transition) density matrices of the ADC calculations were computed via correlated intermediate states. $82[137$ This procedure automatically provides non-trivial values for the deexcitation character $P_{h e}$ and the promotion number $p$. In the case of TDDFT, we used full TDDFT rather than the Tamm-Dancoff approximation to obtain non-vanishing $P_{h e}$ values according to Eq. (51). Non-trivial promotion numbers were obtained with TDDFT after solving the Z-vector equation ${ }^{138}$ following related previous work. 63 The charge transfer measures $d_{h \rightarrow e}$ and $d_{\text {exc }}$ were computed using matrix operations employing the matrix representations of the dipole and quadrupole operators. 99 The $\left\langle\rho_{0 I}|\hat{\mathrm{V}}| \rho_{0 I}\right\rangle$ values were computed in a semi-numerical fashion by performing a numerical integration over the transition density $\rho_{0 I}$ multiplied with the analytically computed ESP $\hat{\mathrm{V}} \rho_{0 I}$. A fully analytical evaluation of $\left\langle\rho_{0 I}|\hat{\mathrm{V}}| \rho_{0 I}\right\rangle$ and $\left\langle\gamma_{0 I}\left|r_{12}^{-1}\right| \gamma_{0 I}\right\rangle$ is possible, in principle, but out of the scope of this work. Densities and ESPs were computed directly in Q-Chem with the exception of the conditional densities, which were computed via TheoDORE 140 and orbkit 141 . Plotting of densities and ESPs was carried out using PyMOL $1.8^{142}$ with the help of the external qc_pymol toolkit. 143 The tikz-feynman package $\frac{144}{14}$ was used for plotting the diagrams presented and the the simplewick package ${ }^{145}$ was used for drawing operator contractions.

\section{Results and discussion}

Having developed the theory, we will now endeavour to study how it provides new insight into practical computations. First, we study uracil [Fig. 1 (a)] as a typical small organic molecule with various functional groups. Here, it will be of particular interest to understand the ordering of the lowest singlet and triplet $\pi \pi^{*}$ and $n \pi^{*}$ states. Second, to judge the transferability of the results shown, we will proceed to the polyacenes [Fig. 1 (b)] and study their HOMO-LUMO transitions with a particular focus on the relative energies between the corresponding singlet and triplets states. We conclude by briefly studying octatetraene, which has been studied due to its states of plasmonic nature. 81

\subsection{Uracil: Singlet and triplet $n \pi^{*}$ and $\pi \pi^{*}$ states}

The excitation energies of the lowest singlet and triplet states of uracil were computed using the correlated ab initio method ADC(2). In Tab. 2 we present the excitation energies, the oscillator strengths, the dominant configurations, the four descriptors discussed in Sec. 2.5 as well as the value of the exchange repulsion $\left\langle\rho_{0 I}|\hat{\mathrm{V}}| \rho_{0 I}\right\rangle$, the two charge-transfer measures $d_{h \rightarrow e}$ and $d_{\text {exc }}$, and the correlation coefficient $R_{h e}$. Noting that the ADC(2) energies are in reasonable agreement with literature values $146 \mid 147$ we can proceed to an analysis of the involved wavefunctions. The lowest excited state is a triplet $\pi \pi^{*}$ state at $4.07 \mathrm{eV}$, which is reached by the transition from the HOMO to the LUMO. This is followed by a triplet $n \pi^{*}$ state at $4.70 \mathrm{eV}$ dominated by the HOMO-2 $\rightarrow$ LUMO transition. The corresponding singlet state $\left({ }^{1} n \pi^{*}\right)$ is only slightly higher in energy at $4.95 \mathrm{eV}$. Then another triplet $\pi \pi^{*}$ state is found before the first ${ }^{1} \pi \pi^{*}$ state, the $\mathrm{HOMO} \rightarrow$ LUMO transition located at $5.76 \mathrm{eV}$. At this point two questions come to mind: Why does the HOMO $\rightarrow$ LUMO transition not give rise to the lowest singlet state and why is the ordering $\pi \pi^{*}$ vs $n \pi^{*}$ reversed between the singlet and triplet manifolds? Neither one of these questions can be answered based on the MO energies and we shall endeavour to study them using the methods developed above.

We start by examining the $\Omega$ and $\mathrm{PR}_{\mathrm{NTO}}$ values according to the classification scheme shown in Tab. 1 . The $\Omega$ value is above 0.85 for all states shown meaning that the general scheme developed 
Table 2 Excitation energies $(\Delta E, \mathrm{eV})$, oscillator strengths (f), leading configurations, single-excitation character $(\Omega)$, effective number of configurations $\left(\mathrm{PR}_{\mathrm{NTO}}\right)$, promotion number $(p)$, de-excitation character $\left(P_{h e}\right)$, exchange repulsion $\left(\left\langle\rho_{0 I}|\hat{\mathrm{V}}| \rho_{0 I}\right\rangle\right.$, eV $)$, linear $\left(d_{h \rightarrow e}, \AA\right)$ and root-mean-square $\left(d_{e x c}, \AA\right)$ charge separation for the lowest lying singlet and triplet states of uracil computed at the ADC(2) level of theory.

\begin{tabular}{llllrrrrrrrc}
\hline State & $\Delta E$ & $\mathrm{f}$ & Configurations $^{\mathrm{a}}$ & $\Omega$ & $\mathrm{PR}_{\mathrm{NTO}}$ & \multicolumn{1}{c}{$p$} & $P_{h e}$ & $\left\langle\rho_{0 I}|\hat{\mathrm{V}}| \rho_{0 I}\right\rangle^{\mathrm{b}}$ & $d_{h \rightarrow e}$ & $d_{\text {exc }}$ & $R_{h e}$ \\
\hline $1^{3} \pi \pi^{*}$ & 4.07 & - & $0.95 h l$ & 0.92 & 1.07 & 1.15 & 0.19 & 6.42 & 0.15 & 2.31 & 0.13 \\
$1^{3} n \pi^{*}$ & 4.70 & - & $0.85 h_{2} l+0.27 h_{2} l_{2}+0.23 h_{3} l$ & 0.88 & 1.01 & 1.47 & 0.02 & 0.60 & 1.37 & 2.57 & 0.05 \\
$1^{1} n \pi^{*}$ & 4.95 & 0.00 & $0.87 h_{2} l+0.23 h_{2} l_{2}+0.14 h_{3} l$ & 0.87 & 1.01 & 1.50 & -0.01 & 0.41 & 1.44 & 2.66 & 0.04 \\
$2^{3} \pi \pi^{*}$ & 5.68 & - & $0.79 h_{1} l-0.41 h l_{2}-0.23 h_{1} l_{6}$ & 0.89 & 1.61 & 1.30 & 0.13 & 6.09 & 0.78 & 2.53 & 0.23 \\
$1^{1} \pi \pi^{*}$ & 5.76 & 0.23 & $0.91 h l-0.17 h_{4} l-0.14 h_{1} l_{2}$ & 0.87 & 1.13 & 1.27 & -0.05 & 0.85 & 0.56 & 2.71 & 0.03 \\
\hline
\end{tabular}

a Dominant configurations and coefficients; $h_{x} l_{y}$ refers to the HOMO $-x \rightarrow$ LUMO $+y$ transition.

$\mathrm{b}$ Repulsion between the transition densities. This term only contributes to the energy of singlet states but is formally evaluated here also for the triplets.

(a)

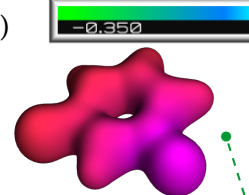

$\mathrm{ESP}, \hat{\mathrm{V}} \rho_{h}$

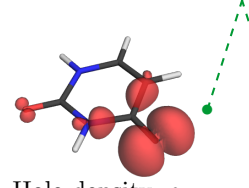

Hole density, $\rho_{h}$

$\triangle$
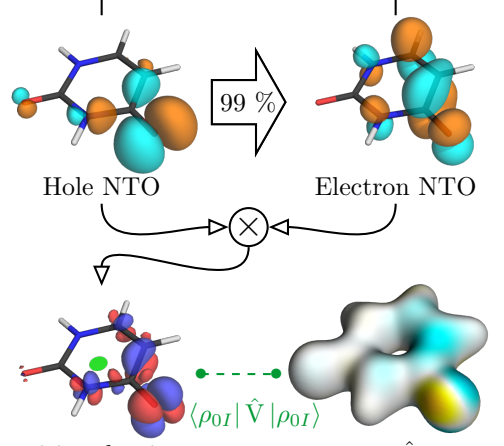

Transition density, $\rho_{0}$

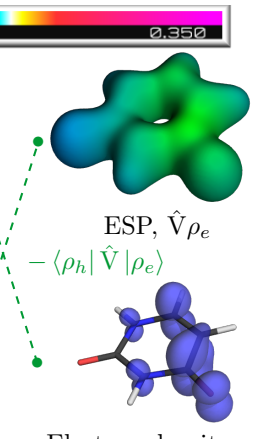

Electron density, $\rho_{e}$

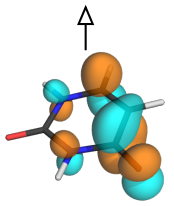

ESP, $\hat{\mathrm{V}} \rho_{0}$ (b)

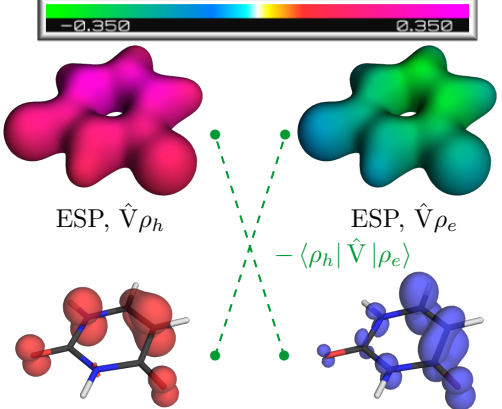

Hole density, $\rho_{h}$
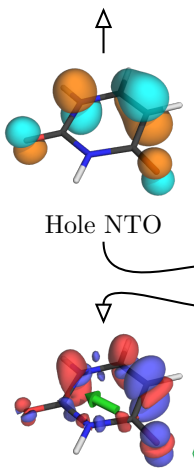

Transition density, $\rho_{0}$

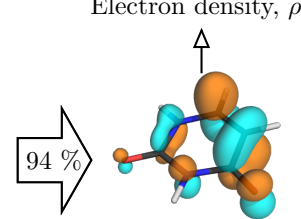

Electron NTO

)

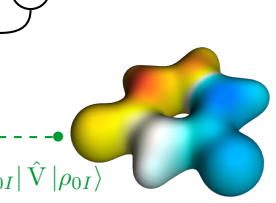

Fig. 6 Analysis of the energetic contributions to (a) the $1^{1} n \pi^{*}$ state, (b) the $1^{1} \pi \pi^{*}$ state, and (c) the $1^{3} \pi \pi^{*}$ state of uracil. The dominant pair of natural transition orbitals and their contribution to the excitation $\left(\lambda_{1} / \Omega\right)$ are shown in the centre of each panel. Above, the hole and electron densities as well as the ESPs induced by them are shown. The Coulomb interaction between electron and hole is indicated as a dashed green line. Below, the transition density and its ESP are shown. The transition dipole moment $\vec{\mu}_{0 I}$ is shown as a green arrow of length $2 \vec{\mu}_{0 I} / e$. Isovalues used: 0.06 for orbitals, 0.005 for densities, 0.05 for the ESP map. Color coding for the ESP maps is shown at the top.

based on CIS is qualitatively correct. In addition, we find that in all cases except for the $2^{3} \pi \pi^{*}$ state the $\mathrm{PR}_{\mathrm{NTO}}$ value is sufficiently close to 1 for the simplified model of Sec. 2.4 to be valid. We choose the $1^{1} n \pi^{*}$, the $1^{1} \pi \pi^{*}$, and the $1^{3} \pi \pi^{*}$ states for a more detailed analysis and present the involved orbital pairs as well as the relevant densities and ESPs in Fig. 6

When the $1^{1} n \pi^{*}$ state is viewed in terms of canonical orbitals, it appears that four orbitals (HOMO-3, HOMO-2, LUMO, LUMO+2) contribute to this state in various combinations. However, once the NTO transformation is applied one finds that $99 \%$ of the 1TDM can be covered with only a single orbital transition, which is presented at the centre of Fig. 6 (a). Accordingly, the $\mathrm{PR}_{\mathrm{NTO}}$ value is very close to 1 . The involved orbitals are a non-bonding orbital localised almost exclusively on oxygen atom $\mathrm{O}_{4}$ (see Fig. 1 for the numbering scheme) and a delocalised $\pi^{*}$ orbital. Comparing these orbitals to the canonical orbitals (Fig. S1) shows that the apparent multiconfigurational character only relates to a change in shape of the orbitals. The hole and electron are both moved more strongly toward the $\mathrm{O}_{4}$ atom when compared to the canonical HOMO-2 and LUMO. This effect may be labelled as "primary orbital relaxation" meaning that the orbitals relevant to the excitation process are different from the Hartree-Fock frontier orbitals representing ionisation and electron attachment. In any case we note that the state is formed by a simple orbital-to-orbital transition in the NTO picture allowing us to proceed by interpreting the last two terms of Eq. (43). First, we need to construct the 
transition density, which is given as the product of the hole and electron NTOs. If the hole is in a $p_{x}$ orbital on oxygen and the electron in a $p_{z}$, then the product will be a $d_{x z}$ orbital on oxygen, as shown in the lower part of Fig. 6 (a). It can be appreciated that a d-orbital possesses no dipole moment (only a quadrupole moment) and therefore makes no contribution to the transition dipole moment. Thus, the transition dipole moments of $n \pi^{*}$ states are generally small. For symmetry reasons, the transition moment is perpendicular to the molecular plane. It is plotted as a green arrow of length $2 \vec{\mu}_{0 I} / e$, which just appears as a green dot in Fig. 6 (a). At the lower right of Fig. 6 (a), we also present the ESP induced by the transition density and map this quantity onto the excited state density. The ESP is close to zero (white) in the largest part and only possesses some slightly positive (yellow) and slightly negative (cyan) areas. Thus, we can appreciate that the self-repulsion of the transition density $\left\langle\rho_{0 I}|\hat{\mathrm{V}}| \rho_{0 I}\right\rangle$ will only be a minor term and indeed we find in Table 2 that its value is only $0.41 \mathrm{eV}$, which is of similar magnitude as the splitting between the singlet and triplet $n \pi^{*}$ states computed at the ADC(2) level $(0.25 \mathrm{eV})$. Above the NTOs, we plot the hole and electron densities. These are constructed as the square of the NTOs and, therefore, possess basically the same shape if the isovalues are chosen accordingly. Their ESPs are shown at the very top. First, it is noted that these ESPs clearly have larger values than the ESP of the transition density meaning that they will give rise to terms of larger magnitude. Second, these ESPs have a very uniform shape showing that the electron-hole attraction will be of similar magnitude for the different valence states.

The singlet and triplet $\pi \pi^{*}$ states are both strongly dominated by the HOMO-LUMO transition but we will find below that their wavefunctions differ in some crucial respects. The primary NTO pair of the $1^{1} \pi \pi^{*}$ state, giving rise to $94 \%$ of its $1 \mathrm{TDM}$, is presented in the centre of Fig. 6 (b). Both NTOs are delocalised over a large part of the molecule. Moving to the top of Fig. 6(b) we find the hole and electron density, which again possess very similar shapes to the original NTOs. The induced ESPs are fairly uniform extending over the whole molecule, similar to Fig. 6(a) showing that the electron-hole attraction will, again, yield a strong but non-specific term. The transition density is constructed as the product of the hole and electron NTOs. It is totally symmetric with respect to reflection at the molecular plane being constructed as a product of two antisymmetric orbitals. One finds that the left part is mostly negative (red) and the right part mostly positive (blue). The transition dipole moment, shown as a green arrow, points from the blue parts to the red parts and one can appreciate that the charge distribution of $\rho_{0 I}$ is quite strongly dipolar and, thus, the state bright. In line with the dipolar nature of the transition density, we also find that its ESP is divided into two areas positive (yellow) on the left and negative (blue) on the right.

On closer inspection, we find that the transition density of the ${ }^{1} \pi \pi^{*}$ state possesses some $\sigma$ contributions, which are seemingly in contradiction to the overall $\pi \pi^{*}$ nature of this state. To investigate this phenomenon in some more detail, the $1^{3} \pi \pi^{*}$ state is discussed as well, see Fig. 6 (c). Its primary NTO pair possesses an almost indistinguishable shape and very similar ampli- tude ( $96 \%$ vs $94 \%$ ) to the $1^{1} \pi \pi^{*}$ state and one might be tempted to conclude that the singlet and triplet wavefunctions are equivalent aside from spin coupling. However, this assessment changes once we compute the transition spin-density. ${ }^{148}$ As opposed to Fig. 6(b), the transition spin-density of the triplet state appears much cleaner in the sense that there are no $\sigma$ contributions. This has two important consequences. First, the formal transition dipole moment of the triplet is increased to 7.1 D whereas it is only $3.3 \mathrm{D}$ for the singlet. Second, the ESP associated to the transition density is much more intense. Indeed we find that the $\left\langle\rho_{0 I}|\hat{\mathrm{V}}| \rho_{0 I}\right\rangle$ term is only $0.85 \mathrm{eV}$ for the singlet while it would amount to $6.42 \mathrm{eV}$ for the triplet. We can interpret this in the following way: The pure HOMO-LUMO transition would give rise to a transition density with a very large self-repulsion and a high energetic penalty. In order to overcome this penalty, a few $\sigma$ contributions are mixed into the wavefunction to partially cancel out the repulsion yielding a lower energy but also darker singlet than the pure HOMO-LUMO transition.

Next, we want to proceed to an analysis of the terms giving rise to the final correlated energies of the $1^{1} n \pi^{*}, 1^{3} n \pi^{*}, 1^{1} \pi \pi^{*}$, and $1^{3} \pi \pi^{*}$ states according to Eqs (43) and (44). For this purpose, we use the hierarchy given by the various orders of the ADC method. The ADC $(0)$ method simply uses orbital energy differences and, therefore, relates to the $\varepsilon_{e}-\varepsilon_{h}$ term. The $\mathrm{ADC}(1)$ energy is equivalent to CIS and reflects the model developed above. The triplet energies only feel the Coulomb term $\left\langle\rho_{h}|\hat{\mathrm{V}}| \rho_{e}\right\rangle$ and we can, thus, use the ADC(1) triplet energies for its estimation. Furthermore, we find the exchange term $\left\langle\rho_{0 I}|\hat{\mathrm{V}}| \rho_{0 I}\right\rangle$ by regarding the singlettriplet splitting. And, finally, the difference between ADC(1) and $\mathrm{ADC}(2)$ is interpreted in terms of secondary orbital relaxation and dynamic correlation. The MO energy gaps are presented in the left-most part of Fig. 7. These grossly overestimate the excitation energies yielding values well above $10 \mathrm{eV}$ and the states move into the expected energy range only after the electron-hole Coulomb attraction is activated. We find that the Coulomb term makes a very strong contribution but also that this term is rather uniform among the states, which is consistent with the fact that it can be effectively hidden inside the DFT orbital energies as discussed at bottom of Sec. 2.4 Including the exchange interaction splits the singlet and triplet states where the effect is much stronger on the $\pi \pi^{*}$ states in line with the above discussion. The inclusion of dynamic correlation tends to lower the gap between the individual states shown here. Fig. 7 also presents the transition dipole moments of the ${ }^{1} \pi \pi^{*}$ states associated to the various models. The crucial observation is that the original "pure" HOMO-LUMO transition would possess a transition moment of $5.7 \mathrm{D}$ and that this value is almost halved once exchange and dynamic correlation are activated. As discussed in the context of Fig. 6) (c) the ADC(2) triplet has an even higher formal transition moment of 7.1 D.

Revisiting Table 2. we find that the $1^{1} n \pi^{*}$ state is characterised by a particularly large promotion number $(p=1.50)$. To understand this effect in more detail, we plot the electron and hole densities $\rho_{e}$ and $\rho_{h}$ in Fig. 8 along with the attachment and detachment densities $\rho_{A}$ and $\rho_{D} . \rho_{e}$ and $\rho_{h}$ represent the primary excitation process whereas $\rho_{A}$ and $\rho_{D}$ also encompass secondary density relaxation effects. To isolate the density relaxation effects, 


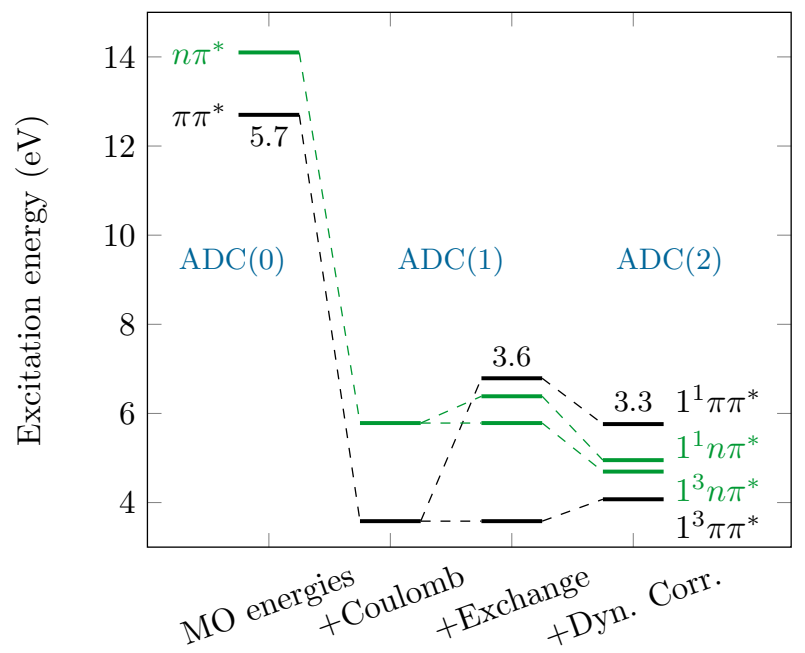

Fig. 7 Energetic contributions, determined via the $A D C(0), A D C(1)$, and $A D C(2)$ methods, giving rise to the energies of the lowest singlet and triplet $n \pi^{*}$ and $\pi \pi^{*}$ states of uracil. The numbers printed correspond to the transition moment (in Debye) of the ${ }^{1} \pi \pi^{*}$ state.

we compute the differences $\rho_{A}-\rho_{e}$ and $\rho_{D}-\rho_{h}$, which are shown at the centre. One can see that $\rho_{A}-\rho_{e}$ covers about the space occupied by $\rho_{h}$ and vice versa. Thus, the secondary relaxation process takes electron density from the area of the excited electron and puts them near the excitation hole. This can be seen as a dielectric screening process, which lowers the energy for separating the primary charges.

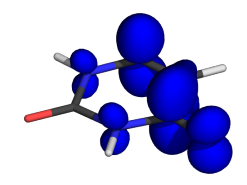

$\rho_{e}$

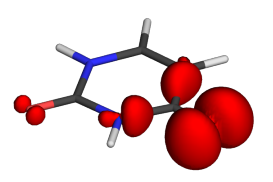

$\rho_{h}$

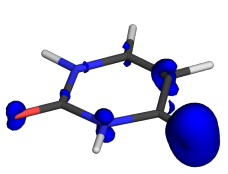

$\rho_{A}-\rho_{e}$

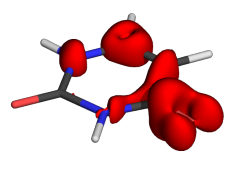

$\rho_{D}-\rho_{h}$

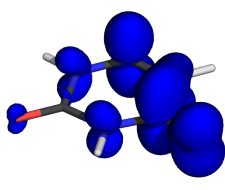

$\rho_{A}$

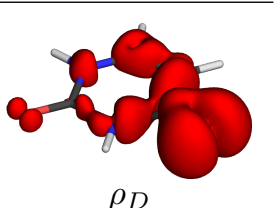

$\rho_{D}$
Fig. 8 Analysis of the $1^{1} n \pi^{*}$ state of uracil. Comparison of the electron and hole densities $\rho_{e}$ and $\rho_{h}$ (left), derived from the 1TDM, with the attachment and detachment densities $\rho_{A}$ and $\rho_{D}$ (right) as derived from the difference density matrix. Their difference, interpreted as the density relaxation, is shown in the centre.

So far, we have restricted the discussion to the four states that can approximately be described by a single NTO transition and have left the $2^{3} \pi \pi^{*}$ state out. This latter state is characterised by a $\mathrm{PR}_{\mathrm{NTO}}$ value of 1.61 , which indicates significant multiconfigurational character. The two pairs of NTOs contributing to the state are shown at the bottom of Fig. 9. There are two pairs of $\pi / \pi^{*}$ orbitals contributing with $75 \%$ and $22 \%$, respectively. We can first proceed to constructing the overall hole and electron densities as shown in Fig. 9 above the NTOs. These generally have a similar shape to the dominant NTOs and no interesting effects are clear at this point. To understand the underlying correlation ef- fects we construct the conditional electron densities according to Eq. (17). $\frac{42}{42}$ The uracil molecule is divided into three fragments as shown in Fig. 1 (a) and the conditional electron densities are computed for the hole constrained to each of the three fragments. The result is shown in the upper part of Fig. 9. In the left column, the probe hole is shown, which is per construction either on the left, centre, or right of the molecule. The conditional electron densities computed for the three possible probe positions are shown in the right column. We find a strong positive correlation between electron and hole and, in particular, we can view the electronhole pair as oscillating between the two $\mathrm{CO}$ bonds. Reviewing the NTOs we find that all NTOs possess at least some contributions on the $\mathrm{CO}$ bonds but with different signs. Their linear combination leads to the observed interference and correlation effects. This discussion is also instructive from an energetic point of view. The excited electron dynamically follows the hole and, thus, allows to maximise their dynamic Coulomb attraction.

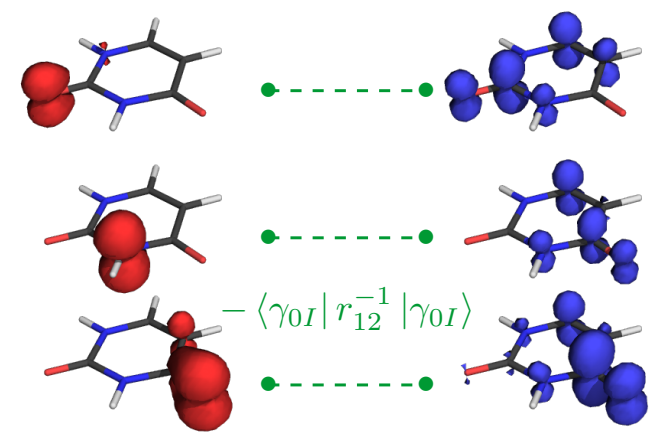

Probe hole

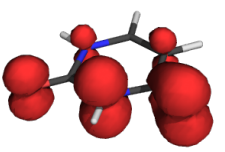

Hole density, $\rho_{h}$
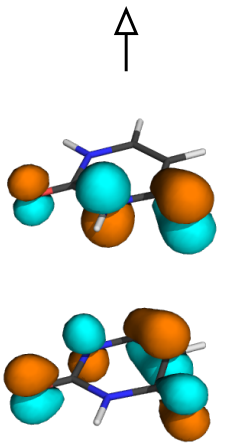

Hole NTOs
Conditional elec. dens.

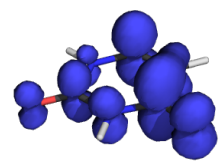

Electron density, $\rho_{e}$
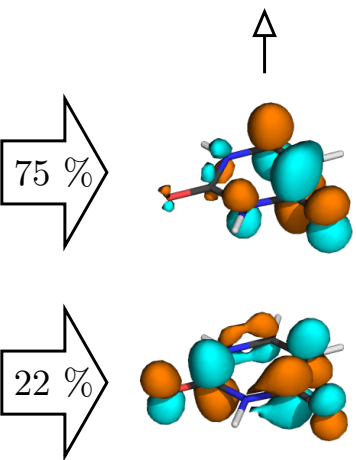

Electron NTOs
Fig. 9 Analysis of the $2^{3} \pi \pi^{*}$ state of uracil. Depiction of correlation effects using conditional densities with the probe hole constrained to three different parts of the molecule. Isovalues used: 0.06 for orbitals, 0.003 for the densities. Conditional densities are plotted as to encompass $75 \%$ of the probability distribution.

We next discuss the charge transfer connected with the different excitations. For this purpose, we look at the linear $\left(d_{h \rightarrow e}\right)$ 
and root-mean-square $\left(d_{\text {exc }}\right)$ electron-hole separations as well as the electron-hole correlation coefficient $\left(R_{h e}\right)$, as shown in Tab.2. The $d_{h \rightarrow e}$ value measures the separation between the centroids of the hole and electron densities. This value is about $1.4 \AA$ for the $n \pi^{*}$ states while it is significantly lower for the $\pi \pi^{*}$ states. This can be understood in the sense that the $\pi$ and $\pi^{*}$ orbitals are all distributed over the molecule fairly evenly while the $n$ orbital is concentrated on one side. Thus, only the transition from the $n$ to the $\pi^{*}$ orbital significantly shifts the electrons on average. As opposed to $d_{h \rightarrow e}$, the $d_{e x c}$ value also considers contributions deriving from the extent of the charge distributions and from correlation effects. ${ }^{99}$ Thus, it is arguably a better approximation to the dynamic electron-hole interaction $\left\langle\gamma_{0 I}\left|r_{12}^{-1}\right| \gamma_{0 I}\right\rangle$. It is found that the $d_{e x c}$ term is fairly uniform between all states considered at $2.5 \pm 0.2 \AA$. This is consistent with the the top row of Fig. 6 highlighting that the Coulomb interactions are fairly uniform for the different states. The correlation coefficient $R_{h e}$ provides a somwhat more sensitive measure $\frac{54 / 70 / 80 \mid 99}{\text { highlighting }}$ whether or not the electron and hole move in a linearly correlated fashion. Viewing Tab. 2, we find that this value is positive for all states considered meaning that dynamic Coulomb attraction dominates over exchange repulsion. The largest $R_{h e}$ value (0.23) is obtained for the $2^{3} \pi \pi^{*}$ state whose wavefunction we have already discussed in Fig. 9. Finally, we compare these measures among the analogous singlet and triplet states, i.e. $1^{1} \pi \pi^{*}$ vs $1^{3} \pi \pi^{*}$ and $1^{1} \pi \pi^{*}$ vs $1^{3} \pi \pi^{*}$. We find that the charge transfer measures $d_{h \rightarrow e}$ and $d_{e x c}$ are generally higher and the correlation coefficient $R_{h e}$ generally lower for the respective singlets. This difference clearly makes sense as the triplet only experiences Coulomb attraction while exchange adds an additional repulsive interaction for the singlets pushing the electron and hole apart.

As a final descriptor, we want to discuss the de-excitation measure $P_{h e}$ as introduced within this work [Eq. (47)]. The absolute $P_{h e}$ values are generally larger for the ${ }^{3} \pi \pi^{*}$ states while they are close to zero for the other states. This can be understood in the sense that the coupling between excitations and de-excitations is mediated by the exchange-like terms shown in Fig. 5 that are related to the exchange repulsion $\left\langle\rho_{0 I}|\hat{V}| \rho_{0 I}\right\rangle$. Thus, the states with the largest $\left\langle\rho_{0 I}|\hat{V}| \rho_{0 I}\right\rangle$ values also experience the largest coupling between excitations and de-excitations. Indeed, the difference between singlet and triplet states with respect to de-excitations and ensuing triplet instabilities have been discussed previously from a more methodological point of view 56 57/96 but this work suggests that this is a physical phenomenon and not related to any specific computational method. Aside from the different magnitude we also obtain different signs for the singlets and triplets as $P_{\text {he }}$ is generally negative in the former and positive for the latter. A detailed discussion of the effect of de-excitations has to be left to the future. Nonetheless, the presented results allow us to postulate that de-excitations do indeed have a non-trivial effect on the relative energies of singlet and triplet states.

Finally, we want to address the question of how much the values of the above descriptors change for different quantum chemical methods. For this purpose, the excited states were recomputed using CASSCF and TDDFT/PBEO and the results are presented in Tab. 3. For both methods we find the same state ordering as for ADC(2). CASSCF places all states within about $0.2 \mathrm{eV}$ of $\operatorname{ADC}(2)$ with the exception of the $1^{1} \pi \pi^{*}$, which also has a grossly overestimated oscillator strength and will be discussed in more detail below. The TDDFT errors are generally somewhat larger, around $0.5 \mathrm{eV}$. Crucially, we find similar trends for most of the descriptors discussed. The $\mathrm{PR}_{\mathrm{NTO}}$ values agree between all three methods in the sense that they are below 1.2 for all states except $2^{3} \pi \pi^{*}$ meaning that the division into simple orbital-to-orbital transitions and multiconfigurational excited states is consistent among the methods. We also find partial agreement among the promotion numbers $p$ measuring the number of rearranged electrons in the sense that they are larger for the $n \pi^{*}$ states than for the $\pi \pi^{*}$ states. Interestingly, also the trends for the newly introduced de-excitation measure $P_{h e}$ agree in magnitude and sign between the three methods. This is a striking observation considering that the de-excitations are included in three completely different ways among the three methods. They are explicitly included via the Y-vector in TDDFT, they appear implicitly in the intermediate state representation ${ }^{82 \mid 137}$ within $\mathrm{ADC}$, and they are computed via the standard density-matrix expressions in CASSCF. We also find similar trends for the $\left\langle\rho_{0 I}|\hat{\mathrm{V}}| \rho_{0 I}\right\rangle$ terms in the sense that they are about 10 times higher for the ${ }^{3} \pi \pi^{*}$ states compared to the others. Furthermore, the CT terms $d_{h \rightarrow e}, d_{e x c}$, and $R_{h e}$ are quite closely matched among the three methods. Comparison of the $\Omega$ values measuring the single-excitation character 61666109 is a bit more troublesome considering that neither $\operatorname{ADC}(2)$ nor TDDFT are able to properly describe double excitations. Interestingly, we find that the $\Omega$ values are even above 1 for TDDFT and a closer look at Eq. (51) shows that they are simply given as

$$
\Omega=\|\mathbf{X}\|^{2}+\|\mathbf{Y}\|^{2}
$$

where $\mathbf{X}$ and $\mathbf{Y}$ are the excitation and de-excitation vectors within TDDFT. On a more fundamental level, this issue is related to the assignment problem of TDDFT, ${ }^{149}$ i.e. it is not clear at all whether it is possible to rigorously construct TDMs and/or wavefunctions from TDDFT. In any case, all the $\Omega$ values are sufficiently close to 1 to exclude any significant double excitation character.

So far, we have left the CASSCF description of the $1^{1} \pi \pi^{*}$ state aside. This state is identified as an ionic state within valence-bond language, a class of states which is notoriously difficult to describe with CASSCF. 47,51 The above discussion provides a clear framework for understanding this problem. The true wavefunction of the $1^{1} \pi \pi^{*}$ state should include $\sigma$-polarisation ${ }^{49 \mid 50}$ to alleviate the energetic penalty deriving from the $\left\langle\rho_{0 I}|\hat{\mathrm{V}}| \rho_{0 I}\right\rangle$ term. However, a standard CASSCF description using only $n$ and $\pi$ orbitals, as employed here, can never describe this effect leaving the energy overestimated. The signature of this problem is also seen in the enhanced oscillator strength of 0.49 for CASSCF deriving from a transition moment of $4.2 \mathrm{D}$, which is intermediate between the pure MO picture and a fully correlated description (Fig.7).

In a more general sense, we want to stress that all the descriptors discussed above are based on density matrices and, thus, ultimately on the wavefunctions. They possess well-defined values for the exact solutions of the electronic Schrödinger equation and are not related to any specific wavefunction model. Even 
Table 3 Excitation energies $(\Delta E, \mathrm{eV})$, oscillator strengths (f), leading configurations and further descriptors computed for the lowest lying singlet and triplet states of uracil computed at the CASSCF and TDDFT/PBEO levels of theory.

\begin{tabular}{|c|c|c|c|c|c|c|c|c|c|c|c|c|}
\hline Method & State & $\Delta E$ & $\mathrm{f}$ & Configurations $^{\mathrm{a}}$ & $\Omega$ & $\mathrm{PR}_{\mathrm{NTO}}$ & $p$ & $P_{h e}$ & $\left\langle\rho_{0 I}|\hat{\mathrm{V}}| \rho_{0 I}\right\rangle$ & $d_{h \rightarrow e}$ & $d_{\text {exc }}$ & $R_{h e}$ \\
\hline \multirow[t]{5}{*}{ CASSCF } & $1^{3} \pi \pi^{*}$ & 4.02 & - & $0.85 h l-0.32 h l_{1}$ & 0.97 & 1.20 & 1.15 & 0.35 & & 0.25 & 2.10 & 0.24 \\
\hline & $1^{3} n \pi^{*}$ & 4.70 & - & $0.87 h_{2} l$ & 0.82 & 1.00 & 1.44 & 0.02 & & 1.11 & 2.23 & 0.00 \\
\hline & $1^{1} n \pi^{*}$ & 5.07 & 0.00 & $0.86 h_{2} l$ & 0.78 & 1.00 & 1.33 & -0.02 & & 1.18 & 2.29 & 0.00 \\
\hline & $2^{3} \pi \pi^{*}$ & 5.93 & - & $-0.77 h_{1} l-0.46 h l_{1}$ & 0.95 & 1.53 & 1.15 & 0.30 & & 0.51 & 2.02 & 0.31 \\
\hline & $1^{1} \pi \pi^{*}$ & 7.27 & 0.49 & $0.83 h l-0.35 c s$ & 0.75 & 1.04 & 1.31 & -0.18 & & 0.60 & 2.73 & -0.04 \\
\hline \multirow[t]{5}{*}{ PBE0 } & $1^{3} \pi \pi^{*}$ & 3.47 & - & $0.97 \mathrm{hl}$ & 1.06 & 1.11 & 1.32 & 0.24 & 7.94 & 0.16 & 2.32 & 0.15 \\
\hline & $1^{3} n \pi^{*}$ & 4.41 & - & $0.92 h_{1} l-0.28 h_{1} l_{1}$ & 1.01 & 1.01 & 1.57 & 0.08 & 0.63 & 1.44 & 2.66 & 0.04 \\
\hline & $1^{1} n \pi^{*}$ & 4.88 & 0.00 & $0.96 h_{1} l$ & 1.00 & 1.01 & 1.61 & -0.03 & 0.35 & 1.55 & 2.80 & 0.03 \\
\hline & $2^{3} \pi \pi^{*}$ & 4.92 & - & $0.81 h_{2} l-0.50 h l_{1}$ & 1.03 & 1.75 & 1.41 & 0.18 & 7.08 & 0.72 & 2.58 & 0.21 \\
\hline & $1^{1} \pi \pi^{*}$ & 5.56 & 0.14 & $0.95 h l$ & 1.03 & 1.17 & 1.42 & -0.01 & 0.61 & 0.71 & 2.87 & -0.02 \\
\hline
\end{tabular}

${ }^{a}$ Dominant configurations and coefficients; $h_{x} l_{y}$ refers to the HOMO- $x \rightarrow$ LUMO $+y$ transition. $c s$ is the closed shell.

more, if we view the wavefunctions as physically meaningful objects rather than just mere mathematical constructs, then it also follows that the descriptors discussed are in a sense physically real. This also suggests that the classification scheme of Tab. 1 is a physically meaningful way to describe electronic excitation processes. Just, as we classify states into $n \pi^{*}$ or $\pi \pi^{*}$ states, even though orbitals are not physically observable, we may also classify them according to the multiconfigurational character, exchange repulsion, de-excitations, or orbital relaxation.

\subsection{Polyacenes: exchange splitting, correlation and de- excitations}

Having discussed the uracil molecule in detail in the previous section, we want to examine the transferability of the observed trends to different molecules. For this purpose, we will study the polyacenes considering their important technological application as versatile organic semiconductors ${ }^{150}$ as well as the fact that they have been studied widely experimentally $151+153$ and computationally. $117 / 154 \quad 156$ Here, we discuss the energies of the lowest singlet and triplet $B_{2 u}$ states. These states are reached via the HOMO-LUMO transition (see also Ref. 155) and are analogous to the $1^{1} \pi \pi^{*}$ and $1^{3} \pi \pi^{*}$ states of uracil as discussed above. The singlet HOMO-LUMO transition is also often referred to as the ${ }^{1} L_{a}$ state. Technologically speaking these two states are relevant because for tetracene and pentacene the $1^{1} B_{2 u}$ state is about twice as high in energy as the $1^{3} B_{2 u}$ state, which allows for the fission of one singlet exciton into two triplet excitons. $23-25$ The consideration of orbital energies is clearly of no use when trying to understand this dramatic difference between two states reached via the same orbital transition and we shall therefore proceed to a more detailed analysis.

The energies and excited-state descriptors, computed for the lowest singlet and triplet $B_{2 u}$ states for the series from naphthalene through pentacene, are presented in Table 4 . We find that the $T_{1}$ energy rapidly decreases along the series. ${ }^{157}$ The gap between $T_{1}$ and $S_{1}$ stays roughly constant at somewhat above $1 \mathrm{eV}$ and, in summary, the $S_{1}$ energy approaches twice the $T_{1}$ energy, which is a prerequisite for singlet fission as discussed above. We start the discussion with the charge transfer character. The linear charge separation $d_{h \rightarrow e}$ vanishes for symmetry reasons and we directly proceed to a discussion of the root-mean-square separation $d_{\text {exc }}$. In analogy to uracil, we find that for each molecule the $d_{\text {exc }}$ value for the singlet is larger than that for the triplet. We also find that the exciton size increases among the series meaning that the state properties are still governed by confinement effects even for pentacene. Previous work suggests that the singlet exciton sizes saturate at larger molecular sizes reaching a universal exciton size limit of about $d_{\text {exc }}=7 \AA . \frac{5498}{4 n}$ In the context of the correlation coefficient $R_{h e}$, we find that its value is consistently around 0.3 for the triplets and significantly smaller for the singlets, again highlighting the stronger influence of Coulomb binding for the triplets. It is also worth pointing out that the low value of $R_{h e}$ for the individual ${ }^{1} L_{a}$ states is consistent with their assignment as weakly correlated " $L_{w}$ " states by Hoffmann et al. 80 The larger $R_{h e}$ values for the triplets also go along with higher $\mathrm{PR}_{\mathrm{NTO}}$ values highlighting the connection between these two measures representing spatial correlation and multiconfigurational character, respectively. Conditional electron densities for the $T_{1}$ state of anthracene were computed to illustrate the underlying correlation effects in some more detail. At the bottom of Fig. 10 the two dominant NTO pairs are shown. The first transition, which corresponds to the HOMO-LUMO transition, accounts for $89 \%$ of the $1 T D M$ with the second pair only contributing 4\%. Nonetheless, we find significant correlation effects. These are shown in the upper part of Fig. 10. Anthracene is divided into three fragments (left, centre and right) and the conditional electron densities for these three fragments are computed according to Eq. (17). On the left, the position of the probe hole is shown while the conditional density is shown on the right. Similarly to Fig. 9, we find a strong correlation between hole and electron and it is clear that this correlation enhances the Coulomb attraction.

Proceeding to the transition densities, we find similar trends as for uracil. The singlet transition densities (Fig. S2) experience significant $\sigma$-polarisation whereas the triplet counterparts (Fig. S3) are derived from pure $\pi$-orbitals. As a consequence, the transition moments $\mu_{0 I}$ increase more than twofold and the exchange repulsion $\left\langle\rho_{0 I}|\hat{\mathrm{V}}| \rho_{0 I}\right\rangle$ about tenfold when moving from the singlets to the triplets (Tab. 4). The effect of the exchange repulsion on transition densities and moments is elucidated in some detail for anthracene in Fig. 11 First, we consider a simple 
Table 4 Excitation energies $(\Delta E, \mathrm{eV})$, transition moments $\left(\mu_{0 I}, \mathrm{D}\right)$, leading configurations and further descriptors computed for the lowest lying singlet and triplet states of the polyacenes computed at the ADC(2)/aug-cc-pVDZ level of theory.

\begin{tabular}{llllcccrrrrr}
\hline Molecule & State & $\Delta E$ & $\mu_{0 I}$ & $\Omega$ & $\mathrm{PR}_{\mathrm{NTO}}$ & $p$ & $P_{h e}$ & $\left\langle\rho_{0 I}|\hat{\mathrm{V}}| \rho_{0 I}\right\rangle$ & $d_{h \rightarrow e}$ & $d_{\text {exc }}$ & $R_{h e}$ \\
\hline Naphthalene & $1^{3} B_{2 u}\left(T_{1}\right)$ & 3.37 & 5.17 & 0.89 & 1.33 & 1.04 & 0.23 & 5.14 & 0.00 & 2.90 & 0.29 \\
& $1^{1} B_{2 u}\left(S_{2}\right)$ & 4.88 & 2.19 & 0.84 & 1.13 & 1.09 & -0.04 & 0.60 & 0.00 & 3.50 & 0.00 \\
\hline Anthracene & $1^{3} B_{2 u}\left(T_{1}\right)$ & 2.44 & 6.41 & 0.86 & 1.23 & 1.07 & 0.22 & 4.60 & 0.00 & 3.43 & 0.28 \\
& $1^{1} B_{2 u}\left(S_{1}\right)$ & 3.71 & 2.52 & 0.82 & 1.08 & 1.13 & -0.07 & 0.52 & 0.00 & 4.07 & 0.04 \\
\hline Tetracene & $1^{3} B_{2 u}\left(T_{1}\right)$ & 1.78 & 7.23 & 0.85 & 1.21 & 1.08 & 0.23 & 4.29 & 0.00 & 3.88 & 0.29 \\
& $1^{1} B_{2 u}\left(S_{1}\right)$ & 2.91 & 2.72 & 0.81 & 1.07 & 1.15 & -0.09 & 0.48 & 0.00 & 4.61 & 0.07 \\
\hline Pentacene & $1^{3} B_{2 u}\left(T_{1}\right)$ & 1.31 & 7.97 & 0.83 & 1.22 & 1.09 & 0.24 & 4.11 & 0.00 & 4.27 & 0.31 \\
& $1^{1} B_{2 u}\left(S_{1}\right)$ & 2.36 & 2.89 & 0.80 & 1.07 & 1.15 & -0.10 & 0.43 & 0.00 & 5.11 & 0.09 \\
\hline
\end{tabular}

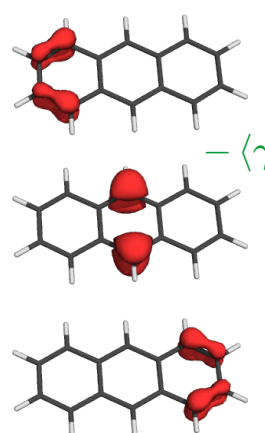

Probe hole

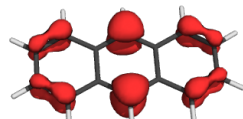

Hole density, $\rho_{h}$

个
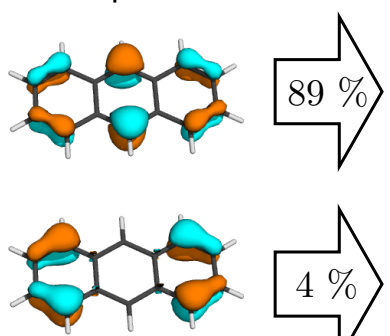

Hole NTOs
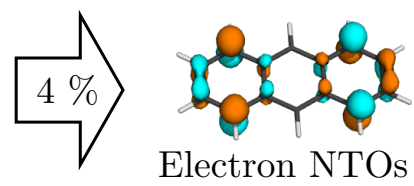

Electron NTOs
Conditional elec. dens.

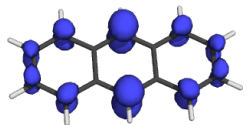

Electron density, $\rho_{e}$

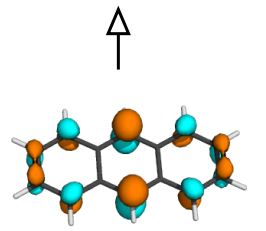
Fig. 10 Depiction of correlation effects in the lowest triplet state $\left(1^{3} B_{2 u}\right)$ of
anthracene using NTOs and conditional densities. Isovalues used: 0.06 for orbitals, 0.003 for the densities, 0.001 for conditional densities.

HOMO-LUMO transition, which is constructed technically via the ADC(0) method. Viewing the HOMO-LUMO transition density in Fig. 11 (a), we find that there is a negative contribution on the upper edge of the molecule encompassing roughly a charge of $0.5 e$ and an analogous positive contribution on the lower edge. Thus, we can estimate the length of the transition moment as

$$
\vec{\mu}_{0 I} \approx 0.5 e \times d_{m o l}
$$

where $e$ is the unit charge and $d_{m o l}$ is the width of the molecule. If we plot the transition moment as $2 \vec{\mu}_{0 I} / e$, this vector should roughly be equal to the width of the molecule, which it as shown in Fig. 11 (a). Introducing $\sigma$-polarisation via the $\operatorname{ADC}(2)$ method roughly halves the transition dipole moment from $5.1 \mathrm{D}$ to $2.5 \mathrm{D}$ as shown in Fig. 11 (b). The experimental transition dipole moment ${ }^{9}$ of $2.8 \mathrm{D}$, agreeing with $\mathrm{ADC}(2)$, is shown in Fig. 11 (c). This agreement clearly highlights the need of using high-level methods like $\mathrm{ADC}(2)$ to obtain accurate transition moments. More strikingly, the relatively low transition moment of anthracene can be seen as an experimental falsification of the HOMO-LUMO picture not requiring any higher level computation. It suffices to know the approximate shapes of the HOMO and LUMO, which follow from symmetry considerations or basic semi-empirical calculations, in order to apply Eq. (53) and deduce that the transition moment of the pure HOMO-LUMO transition should be about $5 \mathrm{D}$. The fact that the measured moment is lower is a direct experimental falsification of the hypothesis that the state is reached via a pure HOMO-LUMO transition.

$$
\text { Transition density }
$$

(a)

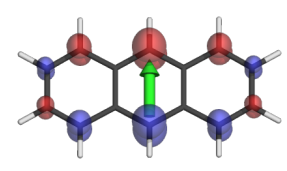

(b)

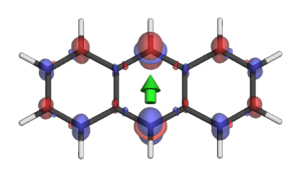

(c)

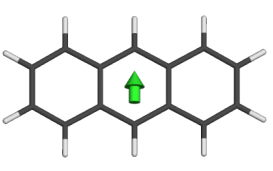

ESP
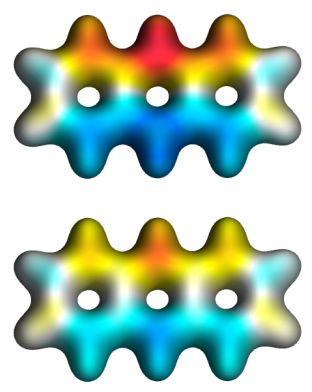

Fig. 11 Depiction of the transition density, transition dipole moment (plotted as a green arrow of length $2 \vec{\mu}_{0 I} / e$ ), and transition ESP for the $1{ }^{1} B_{2 u}$ state of anthracene considering (a) a simple HOMO/LUMO transition and (b) a correlated description. The experimental transition moment is given in (c). Isovalues used: 0.005 for the densities, 0.05 for the ESP map.

Finally, we discuss the de-excitation values $P_{h e}$. Similarly to the $\pi \pi^{*}$ states of uracil these are about 0.2 for the triplet states and slightly below zero for the singlet states. Without going into too much detail we want to stress again that these de-excitations are 
an important component in explaining the energetic difference between the singlet and triplet states and, thus, ultimately the applicability of these molecules for singlet fission.

To summarise, we find three crucial differences between the singlet and triplet wavefunctions. The singlets have enhanced charge transfer, experience $\sigma$-polarisation in their transition densities and have reduced de-excitation character when compared to the singlets. All three phenomena are ultimately responsible for the excitation energies obtained. In the future it will be interesting to examine whether they provide actual handles for tuning the excitation energies. For example, one can ask how the charge transfer is affected by the surrounding medium, if $\sigma$-polarisation is enhanced by polarisable groups and if the de-excitation character is affected by open-shell electrons in the grounds state.

\subsection{Octatetraene: Plasmonic states}

To examine the versatility of the suggested model, we want to examine a physical phenomenon quite different to the above discussion, the presence of plasmonic states in molecules. Plasmonic states in clusters and molecules have received recent attention and significant work has been devoted into finding ways of identifying them within quantum chemistry calculations. 81|104|158 It has been worked out in detail that plasmons can be identified via their dependence on the Coulomb interaction. $81 \mid 104$ We shall build on this work and translate the relations into the nomenclature used here. The previously suggested approach ${ }^{81 \mid 104}$ proceeds by scaling the exchange interaction in Eq. (39) via a parameter $\lambda$ :

$$
\Delta E(\lambda)=\sum_{i a}\left|C_{i a}\right|^{2}\left(\varepsilon_{a}-\varepsilon_{i}\right)+\lambda\left\langle\rho_{0 I}|\hat{\mathrm{V}}| \rho_{0 I}\right\rangle-\left\langle\gamma_{0 I}\left|r_{12}^{-1}\right| \gamma_{0 I}\right\rangle .
$$

After computing the excitation energies for different values of $\lambda$, the ones with the largest changes are identified as plasmons. $\frac{81 \mid 104}{}$ For the purposes of this work, we may directly approximate the slope of $\Delta E$ with respect to $\lambda$ as

$$
\frac{d(\Delta E)}{d \lambda} \approx\left\langle\rho_{0 I}|\hat{\mathrm{V}}| \rho_{0 I}\right\rangle
$$

assuming that the transition density does not have a strong dependence on $\lambda$. Thus, we can directly use the term $\left\langle\rho_{0 I}|\hat{\mathrm{V}}| \rho_{0 I}\right\rangle$ to find the plasmons. We briefly want to discuss the utility of this approach in the case of octatetraene following work by Krauter et al. 81 A summary of the excited states of this system is presented in Tab. 5. The states that were identified as plasmons in Ref. 81 are shown in bold face. Tab. 5 shows that these states are distinguished from the rest by $\left\langle\rho_{0 I}|\hat{\mathrm{V}}| \rho_{0 I}\right\rangle$ values above $1.5 \mathrm{eV}$. This suggests that this value can be used directly rather than following the somewhat more involved scaling approach. Alternatively, it has been shown for metal clusters that plasmons can be identified via excited-state collectivity using what is called here the $\mathrm{PR}_{\mathrm{NTO}}$ descriptor and higher values have been associated with plasmons. ${ }^{158}$ However, this approach does not seem applicable for octatetraene as there is no clear correlation between the plasmon states and $\mathrm{PR}_{\mathrm{NTO}}$.

For comparison, we show the transition densities and ESPs of
Table 5 Excitation energies ( $\Delta E, \mathrm{eV})$, oscillator strengths (f) and further descriptors computed for the lowest lying singlet states of octatetraene computed at the CIS/aug-cc-pVTZ level. ${ }^{a}$

\begin{tabular}{lllccr}
\hline State & $\Delta E$ & $\mathrm{f}$ & $\mathrm{PR}_{\mathrm{NTO}}$ & $\left\langle\rho_{0 I}|\hat{\mathrm{V}}| \rho_{0 I}\right\rangle$ & $d_{\text {exc }}$ \\
\hline $\mathbf{1}^{\mathbf{1}} \mathbf{B}_{\mathbf{u}}$ & $\mathbf{4 . 8 5}$ & $\mathbf{2 . 2 2}$ & 1.17 & $\mathbf{1 . 8 2}$ & 3.64 \\
$\mathbf{2}^{\mathbf{1}} \mathbf{A}_{\mathbf{g}}$ & $\mathbf{7 . 0 5}$ & $\mathbf{0 . 0 0}$ & 2.15 & $\mathbf{2 . 4 0}$ & 2.91 \\
$3^{1} A_{g}$ & 7.65 & 0.00 & 1.97 & 0.20 & 4.75 \\
$\mathbf{2}^{\mathbf{1}} \mathbf{B}_{\mathbf{u}}$ & $\mathbf{8 . 2 5}$ & $\mathbf{0 . 0 8}$ & 2.64 & $\mathbf{2 . 1 5}$ & 2.71 \\
$3^{1} B_{u}$ & 8.79 & 0.03 & 2.16 & 0.45 & 3.70 \\
$4^{1} B_{u}$ & 10.30 & 0.18 & 1.86 & 0.87 & 4.98 \\
\hline
\end{tabular}

a Plasmonic states are highlighted in bold face.

the states discussed in Fig. S4. First, we realise that for all the plasmons the transition densities are located on the atoms rather than the bonds, which allows an identification as ionic states in valence-bond nomenclature. Second, these states possess the same kind of $\sigma$-polarisation as identified for the above molecules. Third, it is observed that the plasmonic states induce intense transition ESPs, which ultimately lead to the large $\left\langle\rho_{0 I}|\hat{\mathrm{V}}| \rho_{0 I}\right\rangle$ values discussed. Thus, the plasmonic states of octatetraene also fit into the general discussion of this work.

\section{Conclusions and Outlook}

The purpose of this Perspective was to lay out a pathway for reaching an understanding of electronic excitation energies that goes beyond the MO picture. What started out with the hope of developing a few simple rules directly applicable to practising chemists ended up as a quite substantial and dense work. There were surprises behind every corner, such as the extensive $\sigma$-polarisation of the transition density, the generality of de-excitations, and the impact of orbital relaxation. Even the seemingly simple case of orbital-to-orbital transitions in organic molecules proved to be much more complex than expected. These challenges also mean that much of the discussion of the even more fascinating case of multiconfigurational states will have to be left to future work.

Despite the challenges mentioned, four distinct physical phenomena, giving rise to effects beyond the MO picture, could be identified: exchange repulsion, dynamic Coulomb attraction, secondary orbital relaxation, and de-excitations. Even more, some preliminary design principles emerge how these phenomena could be used to tune excitation energies and we can speculate about experimentally testable consequences. Exchange repulsion was identified as the Coulomb interaction of the transition density with itself and shown to be a particularly important term for bright singlet states. This term is strongly affected by $\sigma$ polarisation, which lowers singlet-triplet splitting as well as oscillator strength both of which are experimentally observable. In the future it will be of interest to examine whether the amount of $\sigma$ polarisation can be tuned, e.g. by the introduction of polarisable groups. An alternative strategy is concerned with exploiting interference effects in multiconfigurational states as exemplified by the paradigmatic $B_{3 u}^{-}\left(L_{b}\right)$ and $B_{3 u}^{+}\left(B_{b}\right)$ states of naphthalene. 4270 The second term, Coulomb attraction, was shown to be a dynamical effect that is affected by correlation and its magnitude was 
diagnosed by various charge-transfer measures. The main handle one has in terms of affecting the Coulomb interaction is via screening by the solvent. Thirdly, the effect of secondary orbital relaxation was examined and shown to be important for states with strong static charge separation. Finally, we have studied deexcitation character by considering the expectation value of the electron-hole permutation operator and found consistently different values between singlet and triplet states in terms of magnitude and sign.

The wide applicability of the model was exemplified by discussing various molecules and their different types of excited states. In the case of uracil, it was shown why the singlet and triplet $\pi \pi^{*}$ state energies are much more split than their $n \pi^{*}$ counterparts leading to variable state ordering in the singlet and triplet manifolds. Clear differences between the singlet and triplet wavefunctions were shown in terms of $\sigma$-polarisation, charge transfer and de-excitation character. Furthermore, the comparatively low energy of the $2^{3} \pi \pi^{*}$ state of uracil was explained in terms of a dynamically enhanced Coulomb attraction. Studying the singlettriplet splitting in the polyacenes, we found similar wavefunction properties as for the $\pi \pi^{*}$ states of uracil. Specifically, we showed how $\sigma$-polarisation leads to a reduced transition moment of the $1^{1} B_{2 u}$ state of anthracene and we showed that this reduced transition moment is inconsistent with a straightforward HOMO-LUMO transition. A final discussion of octatetraene showed that the presented model is also applicable for characterising plasmonic states in molecules. In the future it will certainly be interesting to extend the discussion to transition metal complexes, which are often characterised by a high density of low-energy singlet and triplet states of varying character.20159|160

The presented discussion has clear consequences on the applicability of modern quantum chemistry methods. Previously, we have shown that the dynamic nature of the Coulomb interaction can deteriorate the reliability of TDDFT even when no apparent CT is present ${ }^{54 / 161}$ and that an overestimation of secondary orbital relaxation effects of $\mathrm{ADC}(2)$ had a negative effect on the computation of the excited states of iridium complexes. ${ }^{55}$ In this work, we have shed new light onto the problem of CASSCF in describing the ionic $1^{1} \pi \pi^{*}$ state of uracil due to the absence of $\sigma$-polarisation. In the future, it will be interesting to assess whether the wide application of the Tamm-Dancoff approximation in TDDFT computations for comparing singlet and triplet energies is justified despite differences in the de-excitation contributions.

In a general sense, we hope that we could convince the reader of the value of analysing excited-state computations beyond the MO picture to reveal the richness of the involved wavefunctions and - as much as wavefunctions are "real" - of physical reality. For this purpose, we advocate an unbiased tool-driven approach keeping eyes open for surprises. Moving our understanding of excited-state electronic structure theory beyond the MO picture is as challenging as it is rewarding and will hopefully be valuable through providing new tools in the design of chromophores and improving our understanding of modern quantum chemistry methods. Ultimately, we hope it will provide a new lens for viewing the world around us.

\section{Conflicts of interest}

There are no conflicts to declare.

\section{Acknowledgements}

The author is grateful to A. Dreuw and S. A. Mewes for various discussions about excited states and orbital energies. The author gratefully acknowledges the use of the 'Lovelace' high performance computing system at Loughborough University.

\section{Notes and references}

1 Q. Zhang, H. Kuwabara, W. J. Potscavage, S. Huang, Y. Hatae, T. Shibata and C. Adachi, J. Am. Chem. Soc., 2014, 136, 18070-18081.

2 Z. Yang, Z. Mao, Z. Xie, Y. Zhang, S. Liu, J. Zhao, J. Xu, Z. Chi and M. P. Aldred, Chem. Soc. Rev., 2017, 46, 915-1016.

3 N. A. Derevyanko, A. A. Ishchenko and A. V. Kulinich, Phys. Chem. Chem. Phys., 2020, 22, 2748-2762.

4 G. Chen, H. Sasabe, T. Igarashi, Z. Hong and J. Kido, J. Mater. Chem. A, 2015, 3, 14517-14534.

5 Y. Shiraishi, S. Kanazawa, Y. Kofuji, H. Sakamoto, S. Ichikawa, S. Tanaka and T. Hirai, Angew. Chemie Int. Ed., 2014, 53, 13454-13459.

6 J. M. Tobin, J. Liu, H. Hayes, M. Demleitner, D. Ellis, V. Arrighi, Z. Xu and F. Vilela, Polym. Chem., 2016, 7, 6662-6670.

7 M. Tromayer, P. Gruber, A. Rosspeintner, A. Ajami, W. Husinsky, F. Plasser, L. González, E. Vauthey, A. Ovsianikov and R. Liska, Sci. Rep., 2018, 8, 17273.

8 C. Castelló Beltrán, E. a. Palmer, B. R. Buckley and F. Iza, Chem. Commun., 2014, 51, 1579-1582.

9 F. Glöcklhofer, A. Rosspeintner, P. Pasitsuparoad, S. Eder, J. Fröhlich, G. Angulo, E. Vauthey and F. Plasser, Mol. Syst. Des. Eng., 2019, 4, 951-961.

10 S. H. Hewitt and S. J. Butler, Chem. Commun., 2018, 54, 6635-6647.

11 Y. Gu, N. Li, G. Shao, K. Wang and B. Zou, J. Phys. Chem. Lett., 2020, 678-682.

12 Y. Wu and W. Zhu, Chem. Soc. Rev., 2013, 42, 2039-2058.

13 B. Holzer, J. Bintinger, D. Lumpi, C. Choi, Y. Kim, B. Stöger, C. Hametner, M. Marchetti-Deschmann, F. Plasser, E. Horkel, I. Kymissis and J. Fröhlich, ChemPhysChem, 2017, 18, 549563.

14 C. Z. Wang, H. Ichiyanagi, K. Sakaguchi, X. Feng, M. R. Elsegood, C. Redshaw and T. Yamato, J. Org. Chem., 2017, 82, 7176-7182.

15 C. Ulbricht, B. Beyer, C. Friebe, A. Winter and U. S. Schubert, Adv. Mater., 2009, 21, 4418-4441.

16 P. A. Scattergood and P. I. Elliott, Dalt. Trans., 2017, 46, 16343-16356.

17 K. Fukui, T. Yonezawa and H. Shingu, J. Chem. Phys., 1952, 20, 722-725.

18 R. Hoffmann, Angew. Chemie Int. Ed. English, 1982, 21, 711724.

19 B. M. Savoie, N. E. Jackson, T. J. Marks and M. A. Ratner, Phys. Chem. Chem. Phys., 2013, 15, 4538-47. 
20 B. J. Powell, Coord. Chem. Rev., 2015, 295, 46-79.

21 J. L. Brédas, Chem. Mater., 2017, 29, 477-478.

22 F. B. Dias, T. J. Penfold and A. P. Monkman, Methods Appl. Fluoresc., 2017, 5, 012001.

23 M. B. Smith and J. Michl, Chem. Rev., 2010, 110, 6891-936.

24 W.-L. Chan, M. Ligges and X.-Y. Zhu, Nat. Chem., 2012, 4, 840-845.

25 D. N. Congreve, J. Lee, N. J. Thompson, E. Hontz, S. R. Yost, P. D. Reusswig, M. E. Bahlke, S. Reineke, T. Voorhis and M. A. Baldo, Science, 2013, 340, 334-337.

26 P. De Silva, J. Phys. Chem. Lett., 2019, 10, 5674-5679.

27 I. Paci, J. C. Johnson, X. Chen, G. Rana, D. Popović, D. E. David, A. J. Nozik, M. A. Ratner and J. Michl, J. Am. Chem. Soc., 2006, 128, 16546-16553.

28 T. Minami and M. Nakano, J. Phys. Chem. Lett., 2012, 3, $145-150$.

29 R. Nieman, N. J. Silva, A. J. A. Aquino, M. M. Haley and H. Lischka, J. Org. Chem., 2020, acs.joc.9b03308.

30 K. J. Fallon, P. Budden, E. Salvadori, A. M. Ganose, C. N. Savory, L. Eyre, S. Dowland, Q. Ai, S. Goodlett, C. Risko, D. O. Scanlon, C. W. M. Kay, A. Rao, R. H. Friend, A. J. Musser and H. Bronstein, J. Am. Chem. Soc., 2019, 141, 13867-13876.

31 S. Montanaro, A. J. Gillett, S. Feldmann, E. W. Evans, F. Plasser, R. H. Friend and I. A. Wright, PCCP, 2019, 21, 10580-10586.

32 S. Brazovskii and N. Kirova, Chem. Soc. Rev., 2010, 39, 2453.

33 W. Barford, J. Phys. Chem. A, 2013, 117, 2665-71.

34 S. A. Mewes, J.-M. Mewes, F. Plasser and A. Dreuw, Phys. Chem. Chem. Phys., 2016, 18, 2548-2563.

35 B. M. Wong, J. Phys. Chem. C. Nanomater. Interfaces, 2009, 113, 21921-21927.

36 L. Stojanović, S. G. Aziz, R. H. Hilal, F. Plasser, T. A. Niehaus and M. Barbatti, J. Chem. Theory Comput., 2017, 13, 58465860.

37 S. A. Bäppler, F. Plasser, M. Wormit and A. Dreuw, Phys. Rev. A, 2014, 90, 052521.

38 R. Pariser, J. Chem. Phys., 1956, 24, 250-268.

39 K. Schulten, I. Ohmine and M. Karplus, J. Chem. Phys., 1976, 64, 4422-4441.

40 T. Hashimoto, H. Nakano and K. Hirao, J. Chem. Phys., 1996, 104, 6244-6258.

41 J. Gu, W. Wu, D. Danovich, R. Hoffmann, Y. Tsuji and S. Shaik, J. Am. Chem. Soc., 2017, 139, 9302-9316.

42 F. Plasser, ChemPhotoChem, 2019, 3, 702-706.

43 S. Grimme and M. Parac, ChemPhysChem, 2003, 4, 292-5.

44 R. M. Richard and J. M. Herbert, J. Chem. Theory Comput., 2011, 7, 1296-1306.

45 N. Kuritz, T. Stein, R. Baer and L. Kronik, J. Chem. Theory Comput, 2011, 7, 2408-2415.

46 A. Prlj, M. E. Sandoval-Salinas, D. Casanova, D. Jacquemin and C. Corminboeuf, J. Chem. Theory Comput., 2016, 12, 2652-2660.

47 B. O. Roos, K. Andersson and M. P. Fülscher, Chem. Phys. Lett., 1992, 192, 5-13.
48 W. T. Borden and E. R. Davidson, Acc. Chem. Res., 1996, 29, 67-75.

49 M. Boggio-Pasqua, M. J. Bearpark, M. Klene and M. A. Robb, J. Chem. Phys., 2004, 120, 7849-7860.

50 C. Angeli, J. Comput. Chem., 2009, 30, 1319-1333.

51 H. Lischka, D. Nachtigallová, A. J. A. Aquino, P. Szalay, F. Plasser, F. B. C. Machado and M. Barbatti, Chem. Rev., 2018, 118, 7293-7361.

52 A. Dreuw, J. L. Weisman and M. Head-Gordon, J. Chem. Phys., 2003, 119, 2943-2946.

53 S. Tretiak, K. Igumenshchev and V. Y. Chernyak, Phys. Rev. $B, 2005,71,33201$.

54 S. A. Mewes, F. Plasser and A. Dreuw, J. Phys. Chem. Lett., 2017, 8, 1205-1210.

55 F. Plasser and A. Dreuw, J. Phys. Chem. A, 2015, 119, 10231026.

56 M. J. Peach and D. J. Tozer, J. Phys. Chem. A, 2012, 116, 9783-9789.

57 M. J. Peach, N. Warner and D. J. Tozer, Mol. Phys., 2013, 111, 1271-1274.

58 D. Jacquemin, I. Duchemin, A. Blondel and X. Blase, J. Chem. Theory Comput., 2017, 13, 767-783.

59 M. Head-Gordon, A. M. Grana, D. Maurice and C. A. White, J. Chem. Phys., 1995, 99, 14261-14270.

60 R. L. Martin, J. Chem. Phys., 2003, 118, 4775-4777.

61 F. Plasser, M. Wormit and A. Dreuw, J. Chem. Phys., 2014, 141, 024106.

62 M. J. G. Peach, P. Benfield, T. Helgaker and D. J. Tozer, J. Chem. Phys., 2008, 128, 44118.

63 E. Ronca, C. Angeli, L. Belpassi, F. D. Angelis, F. Tarantelli and M. Pastore, J. Chem. Theory Comput., 2014, 10, 4014 4024.

64 T. Etienne, X. Assfeld and A. Monari, J. Chem. Theory Comput., 2014, 10, 3906-3914.

65 C. Adamo, T. Le Bahers, M. Savarese, L. Wilbraham, G. Garcia, R. Fukuda, M. Ehara, N. Rega and I. Ciofini, Coord. Chem. Rev., 2015, 304-305, 166-178.

66 S. Matsika, X. Feng, A. V. Luzanov and A. I. Krylov, J. Phys. Chem. A, 2014, 118, 11943-55.

67 G. M. J. Barca, A. T. B. Gilbert and P. M. W. Gill, J. Chem. Theory Comput., 2018, 14, 9-13.

68 A. V. Luzanov and O. V. Prezhdo, Mol. Phys., 2007, 105, 2879-2891.

69 K. Boguslawski, P. Tecmer, O. Legeza and M. Reiher, J. Phys. Chem. Lett., 2012, 3, 3129-3135.

70 F. Plasser, J. Chem. Phys., 2016, 144, 194107.

71 C. J. Stein and M. Reiher, Mol. Phys., 2017, 115, 2110-2119.

72 E. Zojer, P. Buchacher, F. Wudl, J. Cornil, J. P. Calbert, J. L. Brédas and G. Leising, J. Chem. Phys., 2000, 113, 1000210012.

73 J. Rissler, H. Bässler, F. Gebhard and P. Schwerdtfeger, Phys. Rev. B, 2001, 64, 045122.

74 S. Tretiak and S. Mukamel, Chem. Rev., 2002, 102, 3171- 
3212.

75 J. Cornil, I. Gueli, A. Dkhissi, J. C. Sancho-Garcia, E. Hennebicq, J. P. Calbert, V. Lemaur, D. Beljonne and J. L. Brédas, J. Chem. Phys., 2003, 118, 6615-6623.

76 F. Plasser and H. Lischka, J. Chem. Theory Comput., 2012, 8, 2777-2789.

77 Y. Li and C. A. Ullrich, J. Chem. Theory Comput., 2015, 11, 5838-5852.

78 J. J. Nogueira, F. Plasser and L. González, Chem. Sci., 2017, 8, 5682-5691.

79 R. Valero, Á. Morales-García and F. Illas, Phys. Chem. Chem. Phys., 2020, 22, 3017-3029.

80 M. Hoffmann, S. A. Mewes, S. Wieland, C. Popp and A. Dreuw, J. Phys. Chem. Lett., 2019, 10, 6112-6117.

81 C. M. Krauter, S. Bernadotte, C. R. Jacob, M. Pernpointner and A. Dreuw, J. Phys. Chem. C, 2015, 119, 24564-24573.

82 J. Schirmer, Phys. Rev. A, 1982, 26, 2395-2416.

83 C. Hattig, Advances in Quantum Chemistry, Vol 50, 2005, 50, 37-60.

84 G. Onida, L. Reining and A. Rubio, Rev. Mod. Phys., 2002, 74, 601-659.

85 K. Krause and W. Klopper, J. Comput. Chem., 2017, 38, 383388.

86 G. C. Wick, Phys. Rev., 1950, 80, 1950.

87 T. Etienne, 2018.

88 I. Shavitt and R. J. Bartlett, Many-Body Methods in Chemistry and Physics, Cambridge University Press, Cambridge, 2009.

89 A. Szabo and N. S. Ostlund, Modern Quantum Chemistry, Dover Publications, Inc., First Edit edn, 1996.

90 A. V. Luzanov and O. A. Zhikol, Int. J. Quantum Chem., 2010, 110, 902-924.

91 K. D. Nanda and A. I. Krylov, J. Phys. Chem. Lett., 2017, 8, 3256-3265.

92 A. Acharya, S. Chaudhuri and V. S. Batista, J. Chem. Theory Comput., 2018, 14, 867-876.

93 T. Helgaker, P. Jorgensen and J. Olsen, Molecular ElectronicStructure Theory, Wiley, 2000.

94 In Fig. 4 we use the common convention of plotting the sum of the electronic and nuclear ESPs together, i.e. the plotted figure formally contains also the one-electron Coulomb terms.

95 T. Tsuneda and K. Hirao, J. Chem. Phys., 2014, 140, 18A513.

96 A. Dreuw and M. Head-Gordon, Chem. Rev., 2005, 105, 4009-4037.

97 J. Schirmer, Many-Body Methods for Atoms, Molecules and Clusters, Springer International Publishing, Cham, 2018, vol. 94.

98 S. Kraner, R. Scholz, F. Plasser, C. Koerner and K. Leo, J. Chem. Phys., 2015, 143, 244905.

99 F. Plasser, B. Thomitzni, S. A. Bäppler, J. Wenzel, D. R. Rehn, M. Wormit and A. Dreuw, J. Comp. Chem., 2015, 36, 16091620.

100 M. Casida and M. Huix-Rotllant, Annu. Rev. Phys. Chem., 2012, 63, 287-323.
101 For completeness, it should be noted here that the simple relation between the energy and the 1TDM only holds for CIS while the 1TDM takes a somewhat different form in CCS and ADC(1).

102 E. C. Lim and A. L. L. East, J. Chem. Phys., 2000, 113, 89818994.

103 T. Förster, Discuss. Faraday Soc., 1959, 27, 7-17.

104 S. Bernadotte, F. Evers and C. R. Jacob, J. Phys. Chem. C, 2013, 117, 1863-1878.

105 M. Rohlfing and S. G. Louie, Phys. Rev. Lett., 1998, 81, 2312 2315.

106 T. Yanai, D. P. Tew and N. C. Handy, Chem. Phys. Lett., 2004, 393, 51-57.

107 M. S. Hybertsen and S. G. Louie, Phys. Rev. B, 1986, 34, 5390-5413.

108 P. E. Bloechl, S. Lebègue, M. Alouani, P. E. Bloechl, S. Lebègue, M. Alouani, B. Arnaud, B. Arnaud and B. Arnaud, Phys. Rev. B - Condens. Matter Mater. Phys., 2003, 67, 1-10.

109 F. Plasser, S. A. Bäppler, M. Wormit and A. Dreuw, J. Chem. Phys., 2014, 141, 024107.

110 R. Van Meer, O. V. Gritsenko and E. J. Baerends, J. Chem. Theory Comput., 2014, 10, 4432-4441.

111 R. G. Parr and W. Yang, Annu. Rev. Phys. Chem., 1995, 46, 701-728.

112 I. Fernández Galván, Private communication, 2019.

113 F. Plasser, S. A. Mewes, A. Dreuw and L. González, J. Chem. Theory Comput., 2017, 13, 5343-5353.

114 K. Takatsuka, T. Fueno and K. Yamaguchi, Theor. Chem. Acc., 1978, 183, 175-183.

115 V. N. Staroverov and E. R. Davidson, Chem. Phys. Lett., 2000, 330, 161-168.

116 M. Head-Gordon, Chem. Phys. Lett., 2003, 372, 508-511.

117 F. Plasser, H. Pašalic, M. H. Gerzabek, F. Libisch, R. Reiter, J. Burgdörfer, T. Müller, R. Shepard and H. Lischka, Angew. Chem., Int. Ed., 2013, 52, 2581-2584.

118 E. Ramos-Cordoba, P. Salvador and E. Matito, Phys. Chem. Chem. Phys., 2016, 18, 24015-24023.

119 A. V. Luzanov, F. Plasser, A. Das and H. Lischka, J. Chem. Phys., 2017, 146, 064106.

120 S. A. Mewes, F. Plasser and A. Dreuw, J. Chem. Phys., 2015, 143, 171101.

121 W. Barford and N. Paiboonvorachat, J. Chem. Phys., 2008, 129, 164716.

122 T. Etienne, J. Chem. Phys., 2015, 142, 244103.

123 J. R. Platt, J. Chem. Phys., 1949, 17, 484-495.

124 P. R. Surján, Chem. Phys. Lett., 2007, 439, 393-394.

125 I. Mayer, Chem. Phys. Lett., 2007, 443, 420-425.

126 X. Liu, Q. Ou, E. Alguire and J. E. Subotnik, J. Chem. Phys., 2013, 138, 221105.

127 S. Grimme, J. G. Brandenburg, C. Bannwarth and A. Hansen, J. Chem. Phys., 2015, 143, 054107.

128 A. Dreuw and M. Wormit, WIREs: Comp. Mol. Sci., 2015, 5, 
82-95.

129 C. Adamo and V. Barone, J. Chem. Phys., 1999, 110, 61586170.

130 A. Schafer, H. Horn and R. Ahlrichs, J. Chem. Phys., 1992, 97, 2571-2577.

131 R. A. Kendall, T. H. Dunning Jr. and R. J. Harrison, J. Chem. Phys., 1992, 96, 6796.

132 Y. Shao and et al., Mol. Phys., 2015, 113, 184-215.

133 F. Aquilante and et al., J. Comp. Chem., 2016, 37, 506-541.

134 I. Fdez. Galván, M. Vacher, A. Alavi, C. Angeli, F. Aquilante, J. Autschbach, J. J. Bao, S. I. Bokarev, N. A. Bogdanov, R. K. Carlson, L. F. Chibotaru, J. Creutzberg, N. Dattani, M. G. Delcey, S. S. Dong, A. Dreuw, L. Freitag, L. M. Frutos, L. Gagliardi, F. Gendron, A. Giussani, L. González, G. Grell, M. Guo, C. E. Hoyer, M. Johansson, S. Keller, S. Knecht, G. Kovačević, E. Källman, G. Li Manni, M. Lundberg, Y. Ma, S. Mai, J. P. Malhado, P. A. Malmqvist, P. Marquetand, S. A. Mewes, J. Norell, M. Olivucci, M. Oppel, Q. M. Phung, K. Pierloot, F. Plasser, M. Reiher, A. M. Sand, I. Schapiro, P. Sharma, C. J. Stein, L. K. Sørensen, D. G. Truhlar, M. Ugandi, L. Ungur, A. Valentini, S. Vancoillie, V. Veryazov, O. Weser, T. A. Wesołowski, P.-O. Widmark, S. Wouters, A. Zech, J. P. Zobel and R. Lindh, J. Chem. Theory Comput., 2019, 15, 5925-5964.

135 F. Plasser, M. Wormit, S. A. Mewes, B. Thomitzni and A. Dreuw, LIBWFA: Wave-function analysis tool library for quantum chemical applications; available from https://github.com/libwfa/libwfa.

136 Supporting research data available: Molecular geometries, input/output files of Q-Chem and OpenMolcas. DOI: 10.17028/rd.lboro.11548560.

137 J. Schirmer, Phys. Rev. A, 1991, 43, 4647-4659.

138 N. C. Handy and H. F. Schaefer, J. Chem. Phys., 1984, 81, 5031.

139 M. Pastore, X. Assfeld, E. Mosconi, A. Monari and T. Etienne, J. Chem. Phys., 2017, 147, 024108.

140 F. Plasser, TheoDORE: a package for theoretical density, orbital relaxation, and exciton analysis; available from http://theodore-qc.sourceforge.net.

141 G. Hermann, V. Pohl, J. C. Tremblay, B. Paulus, H. C. Hege and A. Schild, J. Comput. Chem., 2016, 37, 1511-1520.
142 Schrödinger, LLC, The PyMOL Molecular Graphics System, Version 1.8, 2015.

143 F. Plasser, qc_pymol: scripts for using pymol together with quantum chemistry programs; available from https://github.com/felixplasser/qc_pymol.

144 J. P. Ellis, Comput. Phys. Commun., 2017, 210, 103-123.

145 J. Kolorenc, simplewick: Wick contractions in LATEX; available from https://www.ctan.org/pkg/simplewick.

146 C. M. Marian, F. Schneider, M. Kleinschmidt and J. Tatchen, Eur. Phys. J. D, 2002, 20, 357-367.

147 E. Epifanovsky, K. Kowalski, P. D. Fan, M. Valiev, S. Matsika and A. I. Krylov, J. Phys. Chem. A, 2008, 112, 9983-9992.

148 The transition densities in Fig. 6 for the singlets are formally obtained by adding the $\alpha$ and $\beta$-spin whereas they are subtracted for the triplets.

149 M. E. Casida, Recent Advances in Density Functional Methods Part I, World Scientific, Singapore, 1995, pp. 155-192.

150 J. E. Anthony, Angew. Chem., Int. Ed., 2008, 47, 452-483.

151 S. H. Lim, T. G. Bjorklund, F. C. Spano and C. J. Bardeen, Phys. Rev. Lett., 2004, 92, 107402-1.

152 R. Schuster, M. Knupfer and H. Berger, Phys. Rev. Lett., 2007, 98, 98-101.

153 T. Sueyoshi, H. Fukagawa, M. Ono, S. Kera and N. Ueno, Appl. Phys. Lett., 2009, 95, 3-6.

154 B. Hajgató, D. Szieberth, P. Geerlings, F. De Proft and M. S. Deleuze, J. Chem. Phys., 2009, 131, 224321.

155 H. F. Bettinger, C. Tonshoff, M. Doerr and E. Sanchez-Garcia, J. Chem. Theory Comput., 2016, 12, 305-312.

156 J. Lee, D. W. Small, E. Epifanovsky and M. Head-Gordon, J. Chem. Theory Comput., 2017, acs.jctc.6b01092.

157 S. Horn, F. Plasser, T. Müller, F. Libisch, J. Burgdörfer and H. Lischka, Theor. Chem. Acc., 2014, 133, 1511.

158 D. Casanova, J. M. Matxain and J. M. Ugalde, J. Phys. Chem. C, 2016, 120, 12742-12750.

159 P. A. Scattergood, A. Sinopoli and P. I. Elliott, Coord. Chem. Rev., 2017, 350, 136-154.

160 S. Mai, F. Plasser, J. Dorn, M. Fumanal, C. Daniel and L. González, Coord. Chem. Rev., 2018, 361, 74-97.

161 S. A. Mewes, F. Plasser and A. Dreuw, J. Chem. Phys., 2015, 143, 171101. 